\title{
Pulsatile flow in a rotating straight pipe: I. Analysis of the fluid motion inside a nutation fluid damper
}

\author{
U. Lei ${ }^{\mathrm{a})}$ and C. F. Ho \\ Institute of Applied Mechanics, National Taiwan University, Taipei 106, Taiwan, Republic of China
}

(Received 13 January 1992; accepted 22 June 1993)

\begin{abstract}
Rigorous analysis shows that the spin synchronous mode fluid motion inside a nutation fluid damper on board of a spinning satellite can be modeled as an incompressible, laminar pulsatile flow in a circular straight pipe. The pipe rotates with constant angular velocity $\underline{\omega}$ about an axis perpendicular to its own axis. The distance between the rotation axis and the pipe axis is much greater than $a$, the pipe's radius. The flow is driven by a three-dimensional harmonic oscillation of the pipe wall with frequency $\Omega$ and amplitude $w_{0}^{\prime}$, and is governed by three-dimensionless parameters: $R_{\Omega}\left(=\Omega a^{2} / v\right), \Delta(=\omega / \Omega)$, and $A\left(=w_{0}^{\prime} / \Omega a\right)$, where $v$ is the kinematic viscosity of the fluid. Both the asymptotic analysis and the numerical calculation have been carried out for $R_{\Omega}=0.1-1000$ and $\Delta=0-2$ under $A \ll 1$. It is found that the rotating effect increases the energy dissipation significantly in comparison with the result of the pulsatile straight pipe flow in an inertia frame (the previous theory for the nutation damper). For $\Delta=1.5$, the energy dissipation in a rotating pipe flow is 5.43 times that in a "stationary" pipe flow for large $R_{\Omega}$, which agrees with the previous experiment. A steady stream is induced by the convective effect for finite values of $A$. Such steady motion is consisted of axial counter flows together with pairs of counter-rotating vortices in the cross-sectional plane.
\end{abstract}

\section{INTRODUCTION}

Study of this problem is motivated by the analysis of the fluid motion inside a nutation fluid damper on a freely precessing satellite, as shown in Fig. 1. Let $\left(X_{b}, Y_{b}, Z_{b}\right)$ be the Cartesian coordinate system fixed to the satellite with the origin $O$ at its mass center. Assume that the satellite is axisymmetric about the $Z_{b}$ axis, with the axial moment of inertia $J$ greater than the transverse moment of inertia $I$. Here the transverse direction is perpendicular to the $Z_{b}$ axis. The system spins about the symmetric axis for system stabilization and attitude control, and possesses minimum total kinetic energy when the nutation angle, $\theta$, equals zero. When $\theta=0$, the direction of the angular velocity $(\omega)$ coincides with that of the angular momentum $H(=J \omega)$. A transverse impulse of angular momentum $\underline{H}_{1}\left(\left|\underline{H}_{1}\right| \ll|\underline{H}|\right)$ may be applied to the system if one wish to change the pointing direction of the spinning axis, say, from a given direction in space to $O O^{\prime}$ in Fig. 1. In such a case, the magnitude of the angular momentum of the spinning system remains unchanged after the impulsive angular momentum has been applied, but the direction of the angular momentum does change to the $O O^{\prime}$ direction, and does not coincide with the direction of the instantaneous angular velocity. The satellite thus undergoes a gyroscopic motion about the $O O^{\prime}$ axis, and possesses excess energy in comparison with the energy for the purely spinning system when $\theta=0$. Figure 1 sketches an instantaneous picture of such gyroscopic motion with finite $\theta$. As in Carrier and Miles, ${ }^{1}$ the precessing angular velocity $\mu \approx(J / I) \omega \hat{e}^{\prime}$ and the spinning angular velocity about the $Z_{b}$ axis

\footnotetext{
"Author to whom correspondence should be addressed.
}

$\underline{\Omega} \approx(1-J / I) \omega \hat{e}^{\prime \prime}$ provided that $\theta$ is sufficiently less than unity, which occurs in many practical systems. Here, $\hat{e}^{\prime \prime}$ and $\hat{e}^{\prime}$ are the unit vectors along the $O Z_{b}$ and $O O^{\prime}$ directions, respectively. Note that $\underline{\mu}+\underline{\Omega} \approx \underline{\omega}$ for $\theta<1$. For the present study, $J>I, \Omega$ is in the opposite direction of $\underline{\omega}$ and $\underline{\mu}$. Referring to the $\left(X_{b}, Y_{b}, Z_{b}\right)$ coordinates in Fig. 1, the angular momentum $\left(\underline{H}=H \hat{e}^{\prime}\right)$ may be decomposed into two components, one along the spin axis $\left(H_{p} \hat{e}^{\prime \prime}\right)$ and one along the transverse direction $\left(H_{t} \hat{e}_{t}\right)$, where $\hat{e}_{t}$ is the unit vector along the transverse direction. One way to remove the unwanted gyroscopic motion is to install a passive nutation fluid damper on the satellite to transfer energy from the transverse axis (associated with $H_{t}$ ) to the spin axis, and to damp out the excess energy of the gyroscopic system (see Cartwright et al. ${ }^{2}$ ).

A nutation fluid damper is a hollow tube formed into closed shapes, usually into a torus or ring, and filled or partly filled with a fluid with certain viscosity. The fluid filled inside the damper is mercury for many practical dampers. When the damper performs properly, the system undergoes a decaying gyroscopic motion with the nutation angle decays with time. Figure 1 shows a partly filled damper mounted with its axis parallel to the spin axis of the satellite, which is the same as those studied by Alfriend $^{3}$ and Cartwright et al. ${ }^{4}$ Here the axis of the damper is defined as the normal of the plane formed by the centerline of the closed tube which passes through the mass center of the empty damper. The mass center of the empty damper is denoted by $O^{\prime \prime}$ in Fig. 1 and is at a distance $h$ from the mass center of the satellite along the axis of the system. In some systems such as that studied by Bhuta and Koval, ${ }^{5}$ the damper may be mounted with its axis perpen- 


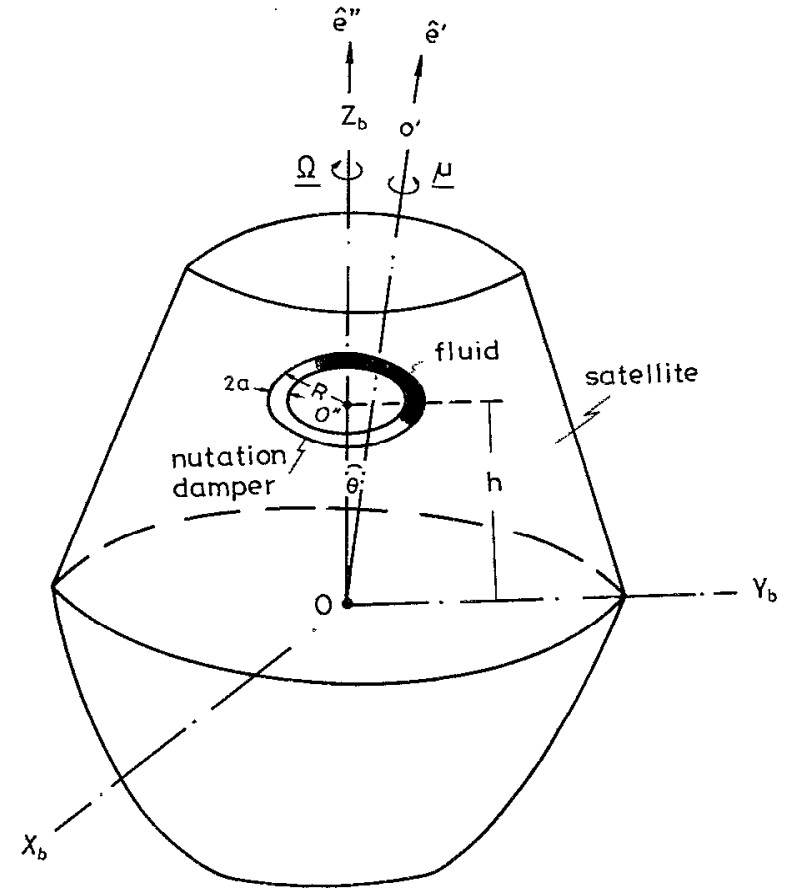

FIG. 1. Sketch of a nutation damper on board of a freely precessing satellite.

dicular to the spin axis of the satellite (see Fig. 2). In the present paper, we shall study the parallel installed damper formed by a circular tube with radius $a$ which is coiled into a circle with radius $R$. Whenever the spin axis of the system docs not coincide with the direction of angular momentum, the system undergoes gyroscopic motion and thus drives the fluid inside the damper via the motion of the damper wall. The fluid motion relative to the wall of the damper dissipate the energy of the system, and hence reduces the nutation angle. Finally, the spin axis coincides with the direction of the constant angular momentum, and the fluid inside the damper undergoes rigid body rotation.

For $\theta \neq 0$, there are three types of fiuid motion in a partly filled mercury damper for the parallel installed case, namely, the nutation synchronous mode, the spin synchro-

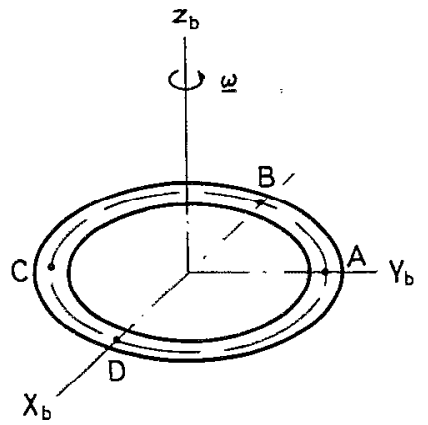

(c)

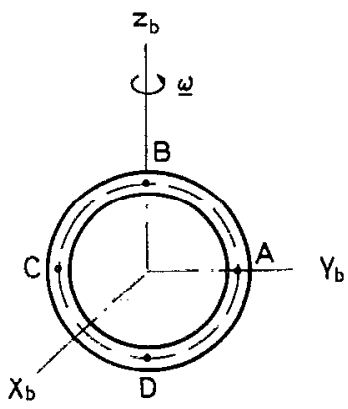

(b)
FIG. 2. Sketch for the installations of the dampers: (a) parallel installed case, the points A, B, C, and D are on the $X_{b} Y_{b}$ plane; (b) perpendicular installed case, the points $\mathrm{A}, \mathrm{B}, \mathrm{C}$, and $\mathrm{D}$ are on the $Y_{b} Z_{b}$ plane.

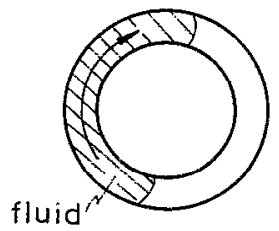

(a) large $\theta$

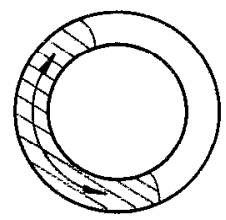

(b) intermediate $\theta$

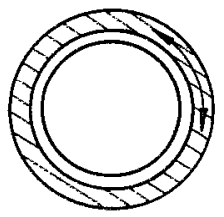

(c) small $\theta$
FIG. 3. Three types of fluid motion in a partly filled nutation fluid damper: (a) nutation synchronous mode, (b) spin synchronous mode, (c) "ring" mode.

nous mode and the "ring" mode, which occur in sequences as $\theta$ decreases (see Fig. 3). The first two modes were named after Cartright et al. ${ }^{4}$ For relative large values of $\theta$, the fluid lumps into a slug and moves unidirectionary with respect to the wall of the damper, and is called the nutation synchronous mode. For sufficiently small $\theta$ (say, less than $0.5^{\circ}$, as suggested by Carrier and Miles ${ }^{1}$ ), the fluid is spread out over the outer part of the damper and formed a continuous ring pattern. Here we call it the ring mode. The fluid in the ring mode oscillates sinusoidally, with respect to the pipe wall due to the nutation effect. Both the nutation mode and the ring mode were first studied by Carrier and Miles ${ }^{1}$ and Miles. ${ }^{6}$ They employed the energy-sink method to determine the decay time constant of the nutation angle, and hence most of their effort is to determine the energy dissipation associated with the relative fluid motion inside the damper. Later, Cartwright et al. ${ }^{4}$ pointed out that in the intermediate range of $\theta$, there exists another mode that the fluid behaves as a slug but oscillates sinusoidally with respect to the wall, and they called it the spin synchronous mode. The fluid motion transits smoothly from the nutation synchronous mode to the spin synchronous mode, and then to the ring mode as the nutation angle decreases. Finally, $\theta$ is reduced to zero and the fluid undergoes rigid body rotation. On some satellites (see Alfriend ${ }^{3}$ ), the center of the damper is offset from the spin axis so that the fluid will always behave as a slug. Alfriend ${ }^{3}$ investigated the dynamics of the satellite for both the nutation synchronous mode and the spin synchronous mode by modeling the fluid as a rigid slug of finite length. The fluid slug is assumed to experience a drag force which is related to the fluid motion inside the damper. The drag 
force is assumed to be proportional to the relative velocity between the slug and the wall. The proportional constant is determined by the shear stress of the fluid motion at the wall for the nutation synchronous mode, and by the average energy dissipation rate associated with the fluid motion for the spin synchronous mode. Based on the above assumptions, Alfriend obtained the time history for the decay of the nutation angle. For the spin synchronous mode, Alfriend compared his theoretical damping constant with Hraster's ${ }^{7}$ experimental findings (see Alfriend ${ }^{8}$ ), and found that the experimental results were 1.85 to 9.97 times the theoretical values for different cases, which implies that the theory predicts a much larger time constant for the decay of the nutation angle. The descrepancy between Alfriend's theory and Hraster's experiment may be due to the following reasons: (1) the fluid mechanics analysis for estimating the rate of energy dissipation, (2) the dynamic model suggested by Alfriend, and (3) the accuracy of the experiments.

The present study try to clear up the above discrepancy between the experiments and the theory for the spin synchronous mode by reconsidering only the fluid mechanics analysis. Alfriend did not study the fluid mechanics in detail, but employed the result of Bhuta and Koval ${ }^{5}$ to determine the damping constant in his dynamic model. Bhuta and Koval ${ }^{5}$ studied a fully filled damper which is installed with its axis perpendicular to the spin axis of the satellite [see Fig. 2(b)]. Based on the situation that the fluid oscillates back and forth, with respect to the wall, and the radius of the tube is much less than the radius of curvature of the damper, Bhuta and Koval modeled the fluid motion inside the damper as pulsatile flow inside an infinite circular straight pipe driven by a harmonic axial oscillation of the pipe wall. The flow is laminar and axisymmetric based on Bhuta and Koval's model. The axial velocity is the only nonzero velocity component, which is simply governed by the diffusion equation and solved via the Laplace transform technique. The average energy dissipation per cycle of the pipe wall oscillation was then obtained and employed to determine the decay time constant for nutation via the energy-sink method. Bhuta and Koval also did some experiments and found that their theoretical predictions on the decay time constant of the nutation angle (which is inverse proportional to the energy dissipation) are $9 \%$ and $15 \%$ higher than the measurements when the rotation Reynolds number $R_{\Omega}$ [defined in Eq. (6)] equals to 8.3 and 7 , respectively.

Alfriend $^{3,8}$ employed Bhuta and Koval's ${ }^{5}$ stationary straight pipe flow model to study the dynamics of a spinning satellite with a partly filled damper, which is mounted with its axis parallel to the spin axis of the satellite [see Fig. 2(a)] when $R_{\Omega}=218-873$ and $\Delta=1.5$ and 2 . Here $\Delta$ is a frequency ratio parameter and is defined in Eq. (6). The adjective "stationary" is employed in this paper to emphasize that the pipe flow in Bhuta and Koval's model occurs in an inertial frame. The reason that Alfriend's theoretical result does not agree with Hraster's ${ }^{7}$ experiment can be study by considering the differences between the stationary straight pipe flow model proposed by Bhuta and Koval and the flow situation in the damper studied by Alfriend. Additional (important) effects can then be included in Bhuta and Koval's model to form a new fluid model. Some of the obvious effects are (1) rotating effect-the pulsate fluid motion inside the damper occurs actually in a noninertial frame (a frame which is mainly rotating with the vector sum of the precessing and the spinning angular velocities of the system) instead in an inertial frame studied by Bhuta and Koval; (2) curvature effect-the damper is a closed curved pipe; (3) end effects of the fluid slug for a partly filled damper; (4) unstable and turbulent effect--the flow inside the damper may be unstable or even turbulent for some of the parameter regimes of the spinning system.

We shall not consider the fourth effect here partly because we do not have a comprehensive understanding on the instability and turbulence of the unsteady rotating flow, and partly because some of the parameter regimes of practical interest are laminar. The analysis of the third effect is difficult because of the existence of the interface; the dynamics of the contact angle had to be considered (see Dussan $^{9}$ ). However, the third effect is expected to be not important on the energy dissipation as soon as the length of the fluid slug is sufficiently greater than the radius (a) of the cross-sectional area of the damper, which is valid for many dampers in practical situation. Both the Coriolis force associated with the first effect and the centrifugal force associated with the second effect may generate a secondary flow in the cross-sectional plane of the damper. The increase of the energy dissipation due to the secondary flow generated by the curvature effect in the pulsating curved pipe flow in an inertial frame is $O(10 \%)$ of the stationary straight pipe result (see Berger et al. ${ }^{10}$ ). Such increase is not important in comparison with the discrepancy in the energy dissipation in Alfriend's case mentioned above, which is $O(100 \%)$ of the stationary straight pipe result. The increase of the energy dissipation due to the secondary flow associatcd with the rotating effect is not clear since the pulsate pipe flow in a rotating frame has not been studied previously, at least up to the authors' knowledge. However, the unidirectional flow in a circular straight pipe rotating with constant angular velocity ( $\underline{\omega})$ about an axis perpendicular to its own axis had been studied quite extensively. Both the experimental measurements by Ito and Nanbu ${ }^{11}$ and the numerical calculations by Lei and $\mathrm{Hsu}^{12}$ showed that the friction factor can be increased up to three times of the corresponding stationary pipe flow results due to the rotating effect for large values of $\omega a^{2} / v$, where $v$ is the kinematic viscosity of the fluid. Hence, it is encouraged to include the first effect in the present study.

In case the end effects of the fluid slug can be neglected, the analysis of the fluid motion for the spin synchronous mode in a partly filled damper is essentially the same as that in a fully filled damper. Let $\dot{\theta}$ be the time rate of change of the nutation angle. When $|\dot{\theta}|<|\underline{\Omega}| \approx|\underline{\mu}|$ (which is valid for the cases studied by Alfriend ${ }^{3,8}$ ), the 


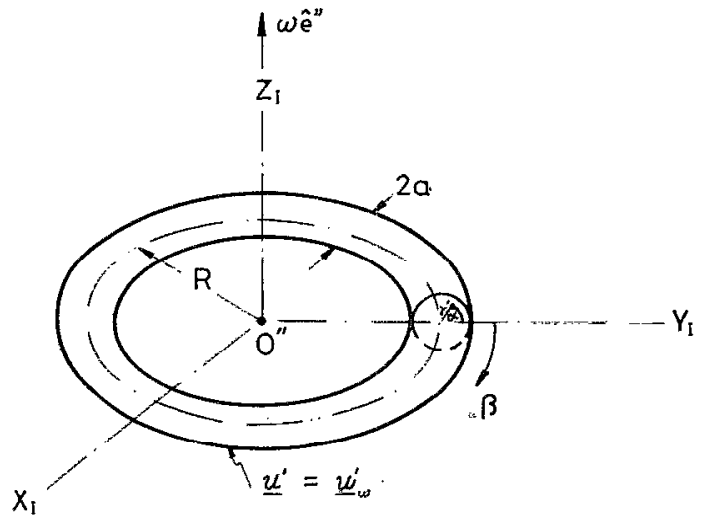

FIG. 4. Configuration of the flow inside a nutation fluid damper. $\left(X_{l}, Y_{I}, Z_{l}\right)$ is an inertial Cartesian coordinates. $\left(r^{\prime}, \alpha, \beta\right)$ is a toroidal coordinates rotating with constant angular velocity $\omega \hat{e}^{\prime \prime}$, where $\hat{e}^{\prime \prime}$ is a unit vector along the $Z_{I}$ direction. Also, $\underline{u}_{w}^{\prime}$ is the prescribed velocity of the pipe wall associated with the nutation effect, which drives the fluid motion inside the curved pipe.

fluid motion inside the damper can be approximated as quasisteady (see Carrier and Miles ${ }^{1}$ ). Referring to the parallel installed case in Fig. 1, the motion of the wall of the damper for $\theta<1$ is mainly rotating with $\mu \cos \theta+\underline{\Omega} \approx \omega \hat{e}^{\prime \prime}$, with superimposed small amplitude oscillation associated with the nutation effect. The quasisteady fluid motion inside a parallel installed, fully filled damper can be approximated as the curved pipe flow problem in a frame rotating with angular velocity $\omega \hat{e}^{\prime \prime}$ in Fig. 4 . A right-handed toroidal coordinates $\left(r^{\prime}, \alpha, \beta\right)$ in the frame rotating with $\omega \hat{e}^{\prime \prime}$ is employed in the present study. The nutation effect is reflected by the eccentric motion of point $O^{\prime \prime}$ around the $O O^{\prime}$ axis in Fig. 1, and is taken into account through a derivation similar to that in Carrier and Miles ${ }^{1}$ as

$$
\begin{gathered}
\underline{u}_{w}^{\prime}=\left[w_{0}^{\prime} \sin \left(\Omega t^{\prime}+\beta\right) \cos \alpha\right] \hat{e}_{r^{\prime}}-\left[w _ { 0 } ^ { \prime } \operatorname { s i n } \left(\Omega t^{\prime}\right.\right. \\
+\beta) \sin \alpha] \hat{e}_{\alpha}-\left[w_{0}^{\prime} \cos \left(\Omega t^{\prime}+\beta\right)\right] \hat{e}_{\beta},
\end{gathered}
$$

which is a three-dimensional oscillation of the pipe wall. Here $w_{0}^{\prime}=\mu h \theta, t^{\prime}$ is the time, and $\hat{e}_{r^{\prime}}, \hat{e}_{\alpha}$, and $\hat{e}_{\beta}$ are the unit vectors along the $r^{\prime}, \alpha$, and $\beta$ directions, respectively. A rigorous analysis in Sec. II will show that the flow in Fig. 4 is governed by four-dimensionless parameters: the curvature parameter $\delta(=a / R)$, the amplitude parameter $A\left[=w_{0}^{\prime} /(\Omega a)\right]$, the frequency ratio $\Delta(=\omega / \Omega)$ and the rotation Reynolds number $R_{\Omega}\left(=\Omega a^{2} / v\right)$. For practical siluations, $\delta=O\left(10^{-1}\right)-O\left(10^{-2}\right)$. Under the condition $\delta<1$, the rotating curved pipe flow problem in Fig. 4 can be reduced to the rotating straight pipe flow problem in Fig. 5 governed only by three parameters: $A, \Delta$, and $R_{\Omega}$. Note that the rotating effect on the fluid motion is much more significant in the parallel installed damper (Alfriend's case) than that in the perpendicular installed damper (Bhuta and Koval's case). This is because $\underline{\omega}$ is always perpendicular to the axial flow for all the positions along the centerline of the damper for the parallel installed case in Fig. 2(a), and thus the Coriolis effect is maximized. On the other hand, the Coriolis force varies along the pipe

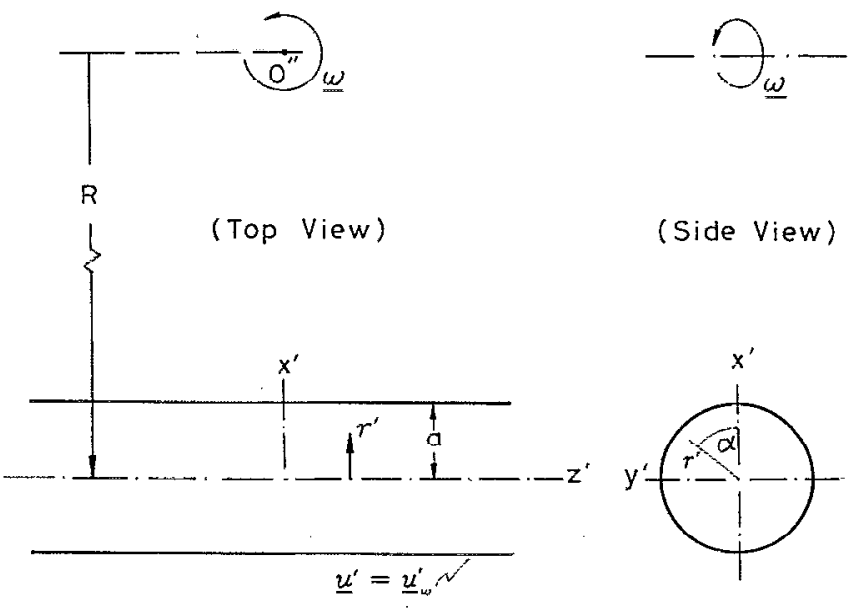

FIG. 5. The rotating straight pipe flow model. $\left(x^{\prime}, y^{\prime}, z^{\prime}\right)$ and $\left(r^{\prime}, \alpha, z^{\prime}\right)$ are the rotating Cartesian and cylindrical coordinates fixed, with respect to the pipe wall. The direction of $\underline{\omega}$ is parallel to the $y^{\prime}$ direction. The distance between the rotation axis and the $y^{\prime}$ axis, $R$, is much greater than the radius of the pipe, $a$.

from zero at location $\mathrm{A}$ (and $\mathrm{C}$ ) to a maximum value at location B (and D) for the perpendicular installed case shown in Fig. 2(b).

The work of the present paper is to analyze the fluid motion in Fig. 5 under the assumption for small values of $A$, with particular emphasis on the energy dissipation associated with the relative fluid motion. The result will be compared with Iraster's ${ }^{7}$ experiment to check if the rotating pipe flow model works. We shall consider the "fully developed" case, which is the case that the flow field is independent of the axial coordinate $\left(z^{\prime}\right.$ in Fig. 5 or $\beta$ in Fig. 4). Beside the above application in the aerospace industry, it is hoped that the nature of a pulsate pipe flow in a rotating frame, in particular the nature of the secondary flow, is of interest to some of the researchers in fluid mechanics.

This paper is the first part of the work on the pulsatile flow in a rotating straight pipe, which includes the formulation of the problem in Sec. II, the linear analysis based on $A \ll 1$ in Sec. III, the asymptotic solutions in Sec. IV, the numerical solutions in Sec. V, a remark on the axial oscillation problem in Sec. VI, and a conclusion in Sec. VII. The cases for $A=O(1)$ have also been studied by solving numerically the unsteady, Navier-Stokes and continuity equations, and the results will be reported separately as Part II of the work on pulsatile flow in a rotating straight pipe.

\section{FORMULATION}

Consider the fluid motion inside a fully filled damper as shown in Fig. 4. The governing equations for the flow of a Newtonian fluid with constant density $\rho$ and kinematic viscosity $v$ in the rotating toroidal coordinates $\left(r^{\prime}, \alpha, \beta\right)$ are 
$\frac{\partial u^{\prime}}{\partial r^{\prime}}+\frac{u^{\prime}}{r^{\prime}}\left(\frac{R+2 r^{\prime} \cos \alpha}{R+r^{\prime} \cos \alpha}\right)+\frac{1}{r^{\prime}} \frac{\partial v^{\prime}}{\partial \alpha}-\frac{v^{\prime} \sin \alpha}{R+r^{\prime} \cos \alpha}+\frac{1}{\left(R+r^{\prime} \cos \alpha\right)} \frac{\partial w^{\prime}}{\partial \beta}=0$,

$$
\begin{aligned}
\frac{\partial u^{\prime}}{\partial t^{\prime}}+u^{\prime} & \frac{\partial u^{\prime}}{\partial r^{\prime}}+\frac{v^{\prime}}{r^{\prime}} \frac{\partial u^{\prime}}{\partial \alpha}+\frac{w^{\prime}}{\left(R+r^{\prime} \cos \alpha\right)} \frac{\partial u^{\prime}}{\partial \beta}-\frac{v^{\prime 2}}{r^{\prime}}-\frac{w^{\prime 2} \cos \alpha}{R-r^{\prime} \cos \alpha}+2 \omega w^{\prime} \cos \alpha \\
= & -\frac{1}{\rho} \frac{\partial p^{\prime *}}{\partial r^{\prime}}-v\left[\left(\frac{1}{r^{\prime}} \frac{\partial}{\partial \alpha}-\frac{\sin \alpha}{R+r^{\prime} \cos \alpha}\right)\left(\frac{\partial v^{\prime}}{\partial r^{\prime}}+\frac{v^{\prime}}{r^{\prime}}-\frac{1}{r^{\prime}} \frac{\partial u^{\prime}}{\partial \alpha}\right)-\frac{1}{\left(R+r^{\prime} \cos \alpha\right)^{2}} \frac{\partial^{2} u^{\prime}}{\partial \beta^{2}}\right. \\
& \left.+\frac{1}{R+r^{\prime} \cos \alpha}\left(\frac{\partial^{2} w^{\prime}}{\partial r^{\prime} \partial \bar{\beta}^{2}}+\frac{\cos \alpha}{\left(R+r^{\prime} \cos \alpha\right)} \frac{\partial w^{\prime}}{\partial \beta}\right)\right],
\end{aligned}
$$

$$
\begin{aligned}
\frac{\partial v^{\prime}}{\partial t^{\prime}}+ & u^{\prime} \frac{\partial v^{\prime}}{\partial r^{\prime}}+\frac{v^{\prime}}{r^{\prime}} \frac{\partial v^{\prime}}{\partial \alpha}+\frac{w^{\prime}}{\left(R+r^{\prime} \cos \alpha\right)} \frac{\partial v^{\prime}}{\partial \beta}+\frac{u^{\prime} v^{\prime}}{r^{\prime}}+\frac{w^{\prime 2} \sin \alpha}{R+r^{\prime} \cos \alpha}-2 \omega w^{\prime} \sin \alpha \\
= & -\frac{1}{\rho r^{\prime}} \frac{\partial p^{\prime *}}{\partial \alpha}+v\left[\frac{1}{\left(R+r^{\prime} \cos \alpha\right)^{2}} \frac{\partial^{2} v^{\prime}}{\partial \beta^{2}} \frac{1}{r^{\prime}\left(R+r^{\prime} \cos \alpha\right)} \frac{\partial^{2} w^{\prime}}{\partial \beta \partial \alpha}+\frac{\sin \alpha}{\left(R+r^{\prime} \cos \alpha\right)^{2}} \frac{\partial w^{\prime}}{\partial \beta}\right. \\
& \left.+\left(\frac{\partial}{\partial r^{\prime}}+\frac{\cos \alpha}{R+r^{\prime} \cos \alpha}\right)\left(\frac{\partial v^{\prime}}{\partial r^{\prime}}+\frac{v^{\prime}}{r^{\prime}}-\frac{1}{r^{\prime}} \frac{\partial u^{\prime}}{\partial \alpha}\right)\right]
\end{aligned}
$$

$$
\begin{aligned}
\frac{\partial w^{\prime}}{\partial t^{\prime}}+ & u^{\prime} \frac{\partial w^{\prime}}{\partial r^{\prime}}+\frac{u^{\prime} w^{\prime} \cos \alpha}{R+r^{\prime} \cos \alpha}+\frac{v^{\prime}}{r^{\prime}} \frac{\partial w^{\prime}}{\partial \alpha}-\frac{v^{\prime} w^{\prime} \sin \alpha}{R+r^{\prime} \cos \alpha}+\frac{w^{\prime}}{\left(R+r^{\prime} \cos \alpha\right)} \frac{\partial w^{\prime}}{\partial \beta}+2 \omega v^{\prime} \sin \alpha-2 \omega u^{\prime} \cos \alpha \\
= & v\left[\left(\frac{\partial}{\partial r^{\prime}}+\frac{1}{r^{\prime}}\right)\left(\frac{\partial w^{\prime}}{\partial r^{\prime}}+\frac{w^{\prime} \cos \alpha}{R+r^{\prime} \cos \alpha}\right)+\frac{1}{r^{\prime 2}} \frac{\partial^{2} w^{\prime}}{\partial \alpha^{2}}-\frac{1}{r^{\prime}} \frac{\partial}{\partial \alpha}\left(\frac{w^{\prime} \sin \alpha}{R+r^{\prime} \cos \alpha}\right)-\left(\frac{\partial}{\partial r^{\prime}}+\frac{1}{r^{\prime}}\right)\left(\frac{1}{\left(R+r^{\prime} \cos \alpha\right)} \frac{\partial u^{\prime}}{\partial \beta}\right)\right. \\
& \left.-\frac{1}{r^{\prime}} \frac{\partial}{\partial \alpha}\left(\frac{1}{\left(R+r^{\prime} \cos \alpha\right)} \frac{\partial v^{\prime}}{\partial \beta}\right)\right]-\frac{1}{\rho\left(R+r^{\prime} \cos \alpha\right)} \frac{\partial p^{\prime *}}{\partial \beta},
\end{aligned}
$$

where $\left(u^{\prime}, v^{\prime}, w^{\prime}\right)$ are the velocity components in the rotating $\left(r^{\prime}, \alpha, \beta\right)$ frame,

$$
p^{\prime *}=p^{\prime}+\rho\left(r^{\prime} R+\frac{r^{\prime 2}}{2} \cos \alpha\right) \omega^{2} \cos \alpha
$$

is the reduced pressure which absorbs the centrifugal force due to the rotation, and $p^{\prime}$ is the pressure. Equations (2a)(2d) are the continuity and Navier-Stokes equations in the rotating toroidal coordinates system. The corresponding equations in an inertial frame can be found from Berger et $a l^{10}$ Note that the gravity has been neglected in the above formulation, which is valid for a satellite operating in the space environment. The boundary conditions for Eqs. (2a) $-(2 d)$ arc

$$
u^{\prime} \hat{e}_{r^{\prime}}+v^{\prime} \hat{e}_{\alpha}+w^{\prime} \hat{e}_{\beta}=\underline{u}_{w}^{\prime} \text { at } r^{\prime}=a,
$$

where $\underline{u}_{w}^{\prime}$ is given by Eq. (1). No initial condition is given here since we shall seek only the long-time response of the flow field, i.e., the quasisteady solution.

The axial flow $\left(w^{\prime}\right)$ and the flow in the cross-sectional plane $\left(u^{\prime}, v^{\prime}\right)$ are coupled to each other through both the Coriolis force and the centrifugal force. The relative importance between these two forces is

$$
\frac{\text { Coriolis force }}{\text { centrifugal force }} \sim \frac{\omega w_{0}^{\prime}}{w_{0}^{\prime 2} / R} \sim \frac{\omega R}{w_{0}^{\prime}} \sim \frac{\omega}{\mu} \frac{R}{h \theta} .
$$

As $\omega / \mu=O(1)$ and $R \gg h \theta$ for most of the systems under consideration, the Coriolis force is much greater than the centrifugal force. On balancing the Coriolis term and the unsteady term in Eqs. (2b) or (2c), one finds that $v^{\prime} \sim u^{\prime} \sim(\omega / \Omega) w^{\prime} \sim w^{\prime}$ for $\omega / \Omega=O(1)$, which is valid for most of the systems of interest. Also the boundary conditions shown in Eq. (1) indicate that $u^{\prime} \sim v^{\prime} \sim w^{\prime}$ $\sim w_{0}^{\prime}$. Hence, Eqs. (2a) $-(2 \mathrm{e})$ are normalized by introducing

$$
\begin{aligned}
& (u, w, w)=\frac{\left(u^{\prime}, v^{\prime}, w^{\prime}\right)}{w_{0}^{\prime}}, \quad r=\frac{r^{\prime}}{a}, \\
& t=t^{\prime} \Omega, \quad p^{*}=\frac{p^{\prime *}}{\rho a \Omega w_{0}^{\prime}} .
\end{aligned}
$$

The resulting dimensionless equations are 
$\frac{\partial u}{\partial r}+\frac{u}{r}\left(\frac{1+2 \delta r \cos \alpha}{1+\delta r \cos \alpha}\right)+\frac{1}{r} \frac{\partial v}{\partial \alpha}-\frac{\delta v \sin \alpha}{1+\delta r \cos \alpha}+\frac{\delta}{(1+\delta r \cos \alpha)} \frac{\partial w}{\partial \beta}=0$

$$
\begin{aligned}
\frac{\partial u}{\partial t}+A & \left(u \frac{\partial u}{\partial r}+\frac{v}{r} \frac{\partial u}{\partial \alpha}+\frac{\delta w}{(1+\delta r \cos \alpha)} \frac{\partial u}{\partial \beta}-\frac{v^{2}}{r}-\frac{\delta w^{2} \cos \alpha}{1+\delta r \cos \alpha}\right)+2 \Delta w \cos \alpha \\
= & -\frac{\partial p^{*}}{\partial r}-\frac{1}{R_{\Omega}}\left[\left(\frac{1}{r} \frac{\partial}{\partial \alpha}-\frac{\delta \sin \alpha}{1+\delta r \cos \alpha}\right)\left(\frac{\partial v}{\partial r}+\frac{v}{r}-\frac{1}{r} \frac{\partial u}{\partial \alpha}\right)\right. \\
& \left.-\frac{\delta^{2}}{(1+\delta r \cos \alpha)^{2}} \frac{\partial^{2} u}{\partial \beta^{2}}+\frac{\delta}{1+\delta r \cos \alpha}\left(\frac{\partial^{2} w}{\partial r \partial \beta}+\frac{\delta \cos \alpha}{(1+\delta r \cos \alpha)} \frac{\partial w}{\partial \beta}\right)\right],
\end{aligned}
$$

$\frac{\partial v}{\partial t}+A\left(u \frac{\partial v}{\partial r}+\frac{v}{r} \frac{\partial v}{\partial \alpha}+\frac{\delta w}{(1+\delta r \cos \alpha)} \frac{\partial v}{\partial \beta}+\frac{u v}{r}+\frac{\delta w^{2} \sin \alpha}{1+\delta r \cos \alpha}\right)-2 \Delta w \sin \alpha$

$$
\begin{aligned}
= & -\frac{1}{r} \frac{\partial p^{*}}{\partial \alpha}+\frac{1}{R_{\Omega}}\left[\frac{\delta^{2}}{(1+2 \delta r \cos \alpha)^{2}} \frac{\partial^{2} v}{\partial \beta^{2}}-\frac{\delta}{r(1+\delta r \cos \alpha)} \frac{\partial^{2} w}{\partial \beta \partial \alpha}+\frac{\delta^{2} \sin \alpha}{(1+\delta r \cos \alpha)^{2}} \frac{\partial w}{\partial \beta}\right. \\
& \left.+\left(\frac{\partial}{\partial r}+\frac{\delta \cos \alpha}{1+\delta r \cos \alpha}\right)\left(\frac{\partial v}{\partial r}+\frac{v}{r}-\frac{1}{r} \frac{\partial u}{\partial \alpha}\right)\right],
\end{aligned}
$$

$\frac{\partial w}{\partial t}+A\left(u \frac{\partial w}{\partial r}+\frac{\delta u w \cos \alpha}{1+\delta r \cos \alpha}+\frac{v}{r} \frac{\partial w}{\partial \alpha}-\frac{\delta v w \sin \alpha}{1+\delta r \cos \alpha}+\frac{\delta w}{(1+\delta r \cos \alpha)} \frac{\partial w}{\partial \beta}\right)+2 \Delta v \sin \alpha-2 \Delta u \cos \alpha$

$$
\begin{aligned}
= & \frac{1}{R_{\Omega}}\left[\left(\frac{\partial}{\partial r}+\frac{1}{r}\right)\left(\frac{\partial w}{\partial r}+\frac{\delta w \cos \alpha}{1+\delta r \cos \alpha}\right)+\frac{1}{r^{2}} \frac{\partial^{2} w}{\partial \alpha^{2}}-\frac{1}{r} \frac{\partial}{\partial \alpha}\left(\frac{\delta w \sin \alpha}{1+\delta r \cos \alpha}\right)\right. \\
& \left.-\left(\frac{\partial}{\partial r}+\frac{1}{r}\right)\left(\frac{\delta}{(1+\delta r \cos \alpha)} \frac{\partial u}{\partial \beta}\right)-\frac{1}{r} \frac{\partial}{\partial \alpha}\left(\frac{\delta}{(1+\delta r \cos \alpha)} \frac{\partial v}{\partial \beta}\right)\right]-\frac{\delta}{(1+\delta r \cos \alpha)} \frac{\partial p^{*}}{\partial \beta},
\end{aligned}
$$

with boundary conditions

$$
\begin{aligned}
& u=\sin (t+\beta) \cos \alpha, \quad v=-\sin (t+\beta) \sin \alpha, \\
& w=-\cos (t+\beta) \quad \text { at } r=1,
\end{aligned}
$$

where

$$
\delta=\frac{a}{R}, \quad A=\frac{w_{0}^{\prime}}{\Omega a}, \quad \Delta=\frac{\omega}{\Omega}, \quad R_{\Omega}=\frac{\Omega a^{2}}{v}
$$

are the four dimensionless parameters governing the fluid motion. For practical situations such as those studied by Alfriend, ${ }^{3,8}$

$$
\begin{aligned}
& \delta=O\left(10^{-2}\right)-O\left(10^{-1}\right), \quad A=O\left(10^{-1}\right)-O(1), \\
& \Delta=O(1), \quad R_{\Omega}=O(10)-O\left(10^{3}\right) .
\end{aligned}
$$

As $\delta$ is always of or less than $O\left(10^{-1}\right)$, one may simplify the analysis by setting $\delta \rightarrow 0$. Equations $(5 \mathrm{a})-(5 \mathrm{~d})$ then reduce to

$$
\begin{aligned}
& \frac{\partial u}{\partial r}+\frac{u}{r}+\frac{1}{r} \frac{\partial v}{\partial \alpha}=0 \\
& \frac{\partial u}{\partial t}+A\left(u \frac{\partial u}{\partial r}+\frac{v}{r} \frac{\partial u}{\partial \alpha}-\frac{v^{2}}{r}\right)+2 \Delta w \cos \alpha \\
& \quad=-\frac{\partial p^{*}}{\partial r}-\frac{1}{R_{\Omega}} \frac{1}{r} \frac{\partial}{\partial \alpha}\left(\frac{\partial v}{\partial r}+\frac{v}{r}-\frac{1}{r} \frac{\partial u}{\partial \alpha}\right),
\end{aligned}
$$

$$
\begin{gathered}
\frac{\partial v}{\partial t}+A\left(u \frac{\partial v}{\partial r}+\frac{v}{r} \frac{\partial v}{\partial \alpha}+\frac{u v}{r}\right)-2 \Delta w \sin \alpha \\
=-\frac{1}{r} \frac{\partial p^{*}}{\partial \alpha}+\frac{1}{R_{\Omega}} \frac{\partial}{\partial r}\left(\frac{\partial v}{\partial r}+\frac{v}{r}-\frac{1}{r} \frac{\partial u}{\partial \alpha}\right), \\
\frac{\partial w}{\partial t}+A\left(u \frac{\partial w}{\partial r}+\frac{v}{r} \frac{\partial w}{\partial \alpha}\right)+2 \Delta v \sin \alpha-2 \Delta u \cos \alpha \\
=\frac{1}{R_{\Omega}}\left(\frac{\partial^{2} w}{\partial r^{2}}+\frac{1}{r} \frac{\partial w}{\partial r}+\frac{1}{r^{2}} \frac{\partial^{2} w}{\partial \alpha^{2}}\right) .
\end{gathered}
$$

Equations (7a)-(7d) depict a loosely coiled pipe limit of the flow in Fig. 4. Note that Eqs. (7a)-(7d) are also the "exact" governing equations for the pulsating "fully developed" flow in a straight pipe which lies on a constant rotating table (see Fig. 5), with $w$ representing the velocity component along the $z^{\prime}$ axis. Here the fully developed flow means that the flow is independent of the axial location of the straight pipe. The rotation axis is parallel to the $y^{\prime}$ axis and passes through the point $O^{\prime \prime}$ in Fig. 5. The distance between the axis of the pipe and the rotation axis is $R$, which is much greater than the pipe's radius, $a$. As the flow described by Eqs. (7a)-(7d) is independent of the axial coordinate $z^{\prime}$ in Fig. 5 (or $\beta$ in Fig. 4 for $\delta<1$ ), the boundary conditions for Eqs. (7a)-(7d) that are consistent with Eq. (5e) are

$$
u=\sin t \cos \alpha, \quad v=-\sin t \sin \alpha,
$$




$$
w=-\cos t \quad \text { at } \quad r=1 .
$$

The rotating pipe flow model described by Eqs. (7a)-(7e) and Fig. 5 is a remedy for Bhuta and Koval's ${ }^{5}$ stationary pipe flow model proposed in the present study. The governing equation for the stationary (straight) pipe flow model, according to Bhuta and Koval, ${ }^{5}$ is

$$
\frac{\partial w}{\partial t}=\frac{1}{R_{\Omega}}\left(\frac{\partial^{2} w}{\partial r^{2}}+\frac{1}{r} \frac{\partial w}{\partial r}\right),
$$

subjected to

$$
w(1, t)=0, \quad t<0 ; \quad w(1, t)=-\cos t, \quad t>0 .
$$

The flow is axisymmetric for the stationary straight pipe flow model. On the other hand, one can find that

$$
\begin{aligned}
& u(r, \alpha, t)=u(r, 2 \pi-\alpha, t), \quad v(r, \alpha, t)=-v(r, 2 \pi-\alpha, t) \\
& w(r, \alpha, t)=w(r, 2 \pi-\alpha, t), \quad p^{*}(r, \alpha, t)=p^{*}(r, 2 \pi-\alpha, t)
\end{aligned}
$$

for the rotating straight pipe model by inspecting Eqs. (7a)-(7e). The rest of this paper is to study the rotating pipe flow model under further assumptions.

There are two differences between the stationary pipe flow model and the rotating pipe flow model arising from the rotating effect: (1) The stationary pipe flow model has only the axial velocity component while the rotating pipe flow model is three dimensional even if the rotating pipe flow is driven only by an axial harmonic oscillation of the pipe wall. The axial flow is coupled with the "secondary flow" in the cross-sectional plane through the Coriolis and the convective effects. (2) The flow in the rotating pipe flow model is driven by a three-dimensional harmonic oscillation [see Eq. (7e)] of the pipe wall, while the pipe wall of the stationary pipe flow model performs only an axial harmonic oscillation [see Eq. (8b)]. Note that the radial and the circumferential components of the pipe wall oscillation have no effect on the energy dissipation for the stationary pipe flow model.

Equation (7a) is satisfied automatically by introducing a stream function $\psi$ such that

$$
u=\frac{1}{r} \frac{\partial \psi}{\partial \alpha}, \quad v=-\frac{\partial \psi}{\partial r} .
$$

The pressure gradient terms can also be eliminated by taking cross differentiation of Eqs. (7b) and (7c). The equations of motion then become

$$
\begin{gathered}
\frac{\partial}{\partial t} \nabla^{2} \psi-\frac{1}{R_{\Omega}} \nabla^{2} \nabla^{2} \psi+2 \Delta\left(\frac{\partial w}{\partial r} \sin \alpha+\frac{1}{r} \frac{\partial w}{\partial \alpha} \cos \alpha\right) \\
=A \frac{1}{r} \frac{\partial\left(\psi, \nabla^{2} \psi\right)}{\partial(r, \alpha)}
\end{gathered}
$$

$$
\begin{aligned}
& \frac{\partial w}{\partial t}-\frac{1}{R_{\Omega}} \nabla^{2} w-2 \Delta\left(\frac{1}{r} \frac{\partial \psi}{\partial \alpha} \cos \alpha+\frac{\partial \psi}{\partial r} \sin \alpha\right) \\
& =A \frac{1}{r} \frac{\partial(\psi, w)}{\partial(r, \alpha)},
\end{aligned}
$$

where $\nabla^{2}$ is the Laplacian operator in the $(r, \alpha)$ plane and $\partial(*, *) / \partial(r, \alpha)$ is the Jacobian operator. The boundary conditions for Eqs. (11a) and (11b) can be derived from Eqs. (7e) and (10) as

$$
\begin{aligned}
& \psi=\sin t \sin \alpha, \quad \frac{\partial \psi}{\partial r}=\sin t \sin \alpha, \\
& w=-\cos t \quad \text { at } r=1 .
\end{aligned}
$$

According to Eqs. (11a)-(11c), we have

$$
\psi(r, \alpha, t)=-\psi(r, 2 \pi-\alpha, t), \quad w(r, \alpha, t)=w(r, 2 \pi-\alpha, t) .
$$

The three-dimensional oscillation problem described by Eqs. (11a)-(11c) can be transformed into an equivalent axial oscillation problem by setting

$$
\psi=\bar{\psi}+\psi_{R}, \quad w=\widetilde{w}+w_{R},
$$

where

$$
\psi_{R}=r \sin t \sin \alpha, \quad w_{R}=-2 \Delta \cos t
$$

describes a rigid body motion. By substituting Eq. (13) into Eqs. (11a)-(11c), we obtain

$$
\begin{aligned}
\frac{\partial}{\partial t} \nabla^{2} \tilde{\psi}-\frac{1}{R_{\Omega}} \nabla^{2} \nabla^{2} \tilde{\psi}+2 \Delta\left(\frac{\partial \tilde{w}}{\partial r} \sin \alpha+\frac{1}{r} \frac{\partial \tilde{w}}{\partial \alpha} \cos \alpha\right) \\
=\frac{A}{r}\left(\frac{\partial\left(\tilde{\psi}, \nabla^{2} \tilde{\psi}\right)}{\partial(r, \alpha)}+\frac{\partial\left(\psi_{R}, \nabla^{2} \widetilde{\psi}\right)}{\partial(r, \alpha)}\right) \\
\frac{\partial \tilde{w}}{\partial t}-\frac{1}{R_{\Omega}} \nabla^{2} \tilde{w}-2 \Delta\left(\frac{1}{r} \frac{\partial \tilde{\psi}}{\partial \alpha} \cos \alpha+\frac{\partial \tilde{\psi}}{\partial r} \sin \alpha\right) \\
=\frac{A}{r}\left(\frac{\partial(\tilde{\psi}, \widetilde{w})}{\partial(r, \alpha)}+\frac{\partial\left(\psi_{R}, \widetilde{w}\right)}{\partial(r, \alpha)}\right)
\end{aligned}
$$

with

$$
\begin{aligned}
& \widetilde{\psi}=0, \quad \frac{\partial \tilde{\psi}}{\partial r}=0, \\
& \widetilde{w}=(2 \Delta-1) \cos t \quad \text { at } r=1 .
\end{aligned}
$$

For the equivalent axial oscillation problem described by Eqs. $(15 a)-(15 c)$, the axial velocity component $\widetilde{w}$ will be called the primary flow while the flow in the $(r, \alpha)$ plane associated with $\widetilde{\psi}$ will be called the secondary flow for the later discussion in this paper.

The dimensionless local rate of energy dissipation per unit mass associated with the relative fluid motion in the rotating straight pipe flow model in Fig. 5 is

$$
\begin{aligned}
\Phi= & \frac{\Phi^{\prime}}{v w_{0}^{\prime 2} / a^{2}} \\
= & 2\left\{\left(\frac{\partial u}{\partial r}\right)^{2}+\left[\frac{1}{r}\left(\frac{\partial v}{\partial \alpha}+u\right)\right]^{2}\right\}+\left(\frac{1}{r} \frac{\partial w}{\partial \alpha}\right)^{2}+\left(\frac{\partial w}{\partial r}\right)^{2} \\
& +\left[\frac{1}{r} \frac{\partial u}{\partial \alpha}+r \frac{\partial}{\partial r}\left(\frac{v}{r}\right)\right]^{2} \\
= & 2\left\{\left(\frac{\partial \widetilde{u}}{\partial r}\right)^{2}+\left[\frac{1}{r}\left(\frac{\partial \widetilde{v}}{\partial \alpha}+\tilde{u}\right)\right]^{2}\right\}+\left(\frac{1}{r} \frac{\partial \widetilde{w}}{\partial \alpha}\right)^{2}+\left(\frac{\partial \widetilde{w}}{\partial r}\right)^{2} \\
& +\left[\frac{1}{r} \frac{\partial \widetilde{u}}{\partial \alpha}+r \frac{\partial}{\partial r}\left(\frac{\widetilde{v}}{r}\right)\right]^{2} .
\end{aligned}
$$


The difference between the function $\Phi$ for the rotating straight pipe flow model in Fig. 5 and that for the rotating curved pipe flow model in Fig. 4 is of order $O\left(\delta^{2}\right)$. Both Bhuta and $\mathrm{Koval}^{5}$ and Alfriend ${ }^{3}$ employed a dimensionless function $g$ to characterize the average normalized energy dissipation per cycle of the pipe wall oscillation. For the present rotating pipe flow model under the quasisteady condition, we need only to consider the flow variation during one cycle of the pipe wall ascillation. In terms of the present notation,

$$
\begin{aligned}
g & \equiv g_{R} \\
& =\frac{E^{\prime}}{M^{\prime} w_{0}^{\prime 2}} \\
& =\frac{1}{\pi R_{\Omega}} \int_{0}^{2 \pi} \int_{0}^{2 \pi} \int_{0}^{1} \Phi\left(r, \alpha, t ; R_{\Omega}, \Delta, A\right) r d r d \alpha d t,
\end{aligned}
$$

where $E^{\prime}$ is the energy dissipated per unit axial length of the fluid during one cycle of the pipe wall oscillation, and $M^{\prime}=\rho \pi a^{2}$ is the mass per unit axial length of the fluid in the pipe. Here we employed the subscript " $R$ " for $g$ in Eq. (17) to refer that it is based on the rotating pipe flow model. Also, $g$ is a function of $R_{\Omega}, \Delta$ and $A$ for the present rotating pipe flow model, but is only function of $R_{\Omega}$ for the stationary pipe flow model proposed by Bhuta and Koval (which will be denoted by $g_{S}$ in the later discussion).

The velocity components of the rigid body motion

$$
\begin{aligned}
& u_{R}=\frac{1}{r} \frac{\partial \psi_{R}}{\partial \alpha}=\sin t \cos \alpha, \\
& v_{R}=-\frac{\partial \psi_{R}}{\partial r}=-\sin t \sin \alpha, \quad w_{R}=-2 \Delta \cos t
\end{aligned}
$$

together with the pressure

$$
p_{R}^{*}=\left(4 \Delta^{2}-1\right) r \cos \alpha \cos t+\text { const }
$$

satisfy Eqs. (11a) and (11b). If further $\Delta=0.5$, they also satisfy Eq. (11c). It follows that

$$
\tilde{\psi}=0, \quad \tilde{w}=0
$$

is a solution for Eqs. (15a)-(15c) when $\Delta=0.5$. There is no deformation and, hence, no energy dissipation when $\Delta=0.5$ in the present rotating pipe flow model. For the linear case when $A=0$, the energy dissipation for the rotating pipe flow model is roughly $(2 \Delta-1)^{2}$ times that for the stationary pipe flow model, according to Eqs. (15c) and (8b). Therefore, the energy dissipation predicted by the rotating pipe flow model may be greater or less than that predicted by the stationary pipe flow model, depending on the magnitude of $\Delta$. For the practical cases such as those studied by Alfriend, ${ }^{3,8} \Delta=1.5$ and 2 , and, hence, $(2 \Delta-1)^{2}=4$ and 9 , respectively. The rotating pipe flow model predicts much more energy dissipation in comparing with that predicted by the stationary pipe flow model, and thus agrees better with Hraster's ${ }^{7}$ experiments.

\section{LINEAR ANALYSIS}

In case the wall oscillates with low amplitude but high frequency, the parameter $A$ is small, and we may set

$$
\widetilde{\psi}(r, \alpha, t)=\widetilde{\psi}_{0}(r, \alpha, t)+A \widetilde{\psi}_{1}(r, \alpha, t)+A^{2} \widetilde{\psi}_{2}(r, \alpha, t)+\cdots
$$

and

$$
\widetilde{w}(r, \alpha, t)=\widetilde{w}_{0}(r, \alpha, t)+A \widetilde{w}_{1}(r, \alpha, t)+A^{2} \widetilde{w}_{2}(r, \alpha, t)+\cdots .
$$

The assumption for small values of $A$ is valid for some systems and for most of the systcms at the final stage of nutation decay. By substituting Eqs. (19a) and (19b) into Eqs. (15a)-(15c), and after certain manipulations, we obtain the following systems of linear equations:

$$
\begin{gathered}
\frac{\partial}{\partial t} \nabla^{2} \widetilde{\psi}_{0}-\frac{1}{R_{\Omega}} \nabla^{2} \nabla^{2} \tilde{\psi}_{0} \\
=-2 \Delta\left(\frac{\partial \widetilde{w}_{0}}{\partial r} \sin \alpha+\frac{1}{r} \frac{\partial \widetilde{w}_{0}}{\partial \alpha} \cos \alpha\right), \\
\frac{\partial \widetilde{w}_{0}}{\partial t}-\frac{1}{R_{\Omega}} \nabla^{2} \widetilde{w}_{0}=2 \Delta\left(\frac{1}{r} \frac{\partial \widetilde{\psi}_{0}}{\partial \alpha} \cos \alpha+\frac{\partial \widetilde{\psi}_{0}}{\partial r} \sin \alpha\right),
\end{gathered}
$$

with boundary conditions

$$
\widetilde{\psi}_{0}=0, \quad \frac{\partial \widetilde{\psi}_{0}}{\partial r}=0, \quad \widetilde{w}_{0}=(2 \Delta-1) \cos t \quad \text { at } r=1,
$$

and

$$
\begin{aligned}
\frac{\partial}{\partial t} \nabla^{2} \widetilde{\psi}_{1}-\frac{1}{R_{\Omega}} \nabla^{2} \nabla^{2} \widetilde{\psi}_{1}+2 \Delta\left(\frac{\partial \widetilde{w}_{1}}{\partial r} \sin \alpha+\frac{1}{r} \frac{\partial \widetilde{w}_{1}}{\partial \alpha} \cos \alpha\right) \\
=\frac{1}{r}\left(\frac{\partial\left(\widetilde{\psi}_{0}, \nabla^{2} \widetilde{\psi}_{0}\right)}{\partial(r, \alpha)}+\frac{\partial\left(\psi_{R}, \nabla^{2} \widetilde{\psi}_{0}\right)}{\partial(r, \alpha)}\right), \\
\frac{\partial \widetilde{w}_{1}}{\partial t}-\frac{1}{R_{\Omega}} \nabla^{2} \widetilde{w}_{1}-2 \Delta\left(\frac{1}{r} \frac{\partial \widetilde{\psi}_{1}}{\partial \alpha} \cos \alpha+\frac{\partial \widetilde{\psi}_{1}}{\partial r} \sin \alpha\right) \\
=\frac{1}{r}\left(\frac{\partial\left(\widetilde{\psi}_{0}, \widetilde{w}_{0}\right)}{\partial(r, \alpha)}+\frac{\partial\left(\psi_{R}, \widetilde{w}_{0}\right)}{\partial(r, \alpha)}\right),
\end{aligned}
$$

with boundary conditions:

$$
\widetilde{\psi}_{1}=\frac{\partial \widetilde{\psi}_{1}}{\partial r}=\widetilde{w}_{1}=0 \quad \text { at } r=1 .
$$

Recall from Eq. (12) that we have conditions imposed on the line $\alpha=0$ (or $\pi$ ) for the general nonlinear flow governed by Eqs. (11a)-(11c). For the linearized flow quantities $\widetilde{\psi}_{0}, \widetilde{\psi}_{1}, \ldots .$. , and $\widetilde{w}_{0}, \widetilde{w}_{1}, \ldots$. in Eqs. (19a) and (19b), we have

$$
\begin{aligned}
& \widetilde{w}_{0}(r, \alpha, t)=\widetilde{w}_{0}(r, 2 \pi-\alpha, t), \\
& \widetilde{\psi}_{0}(r, \alpha, t)=-\widetilde{\psi}_{0}(r, 2 \pi-\alpha, t),
\end{aligned}
$$




$$
\begin{aligned}
& \widetilde{w}_{1}(r, \alpha, t)=\widetilde{w}_{1}(r, 2 \pi-\alpha, t), \\
& \widetilde{\psi}_{1}(r, \alpha, t)=-\widetilde{\psi}_{1}(r, 2 \pi-\alpha, t),
\end{aligned}
$$

as well as

$$
\begin{aligned}
& \widetilde{w}_{0}(r, \alpha, t)=\widetilde{w}_{0}(r, \pi-\alpha, t), \quad \widetilde{\psi}_{0}(r, \alpha, t)=\widetilde{\psi}_{0}(r, \pi-\alpha, t), \\
& \widetilde{w}_{1}(r, \alpha, t)=-\widetilde{w}_{1}(r, \pi-\alpha, t), \\
& \widetilde{\psi}_{1}(r, \alpha, t)=-\widetilde{\psi}_{1}(r, \pi-\alpha, t)
\end{aligned}
$$

by inspecting Eqs. (20a)-(20c) and (21a)-(21c). Hence, we need to consider only the flow in the first quadrant of Fig. 5 for analyzing the linearized flow field.

Solving Eqs. (20a)-(20c) is equivalent to solving the following complex problem:

$$
\begin{aligned}
\frac{\partial}{\partial t} \nabla^{2} \psi_{0}^{*}-\frac{1}{R_{\Omega}} \nabla^{2} \nabla^{2} \psi_{0}^{*} \\
=-2 \Delta\left(\frac{\partial w_{0}^{*}}{\partial r} \sin \alpha+\frac{1}{r} \frac{\partial w_{0}^{*}}{\partial \alpha} \cos \alpha\right),
\end{aligned}
$$

$\frac{\partial w_{0}^{*}}{\partial t}-\frac{1}{R_{\Omega}} \nabla^{2} w_{0}^{*}=2 \Delta\left(\frac{1}{r} \frac{\partial \psi_{0}^{*}}{\partial \alpha} \cos \alpha+\frac{\partial \psi_{0}^{*}}{\partial r} \sin \alpha\right)$,

with

$$
\psi_{0}^{*}=0, \quad \frac{\partial \psi_{0}^{*}}{\partial r}=0, \quad w_{0}^{*}=(2 \Delta-1) e^{i t} \quad \text { at } r=1,
$$

where $\psi_{0}^{*}$ and $w_{0}^{*}$ are complex functions of $r, \alpha$, and $t$, and

$$
\widetilde{\psi}_{0}=\operatorname{Re}\left(\psi_{0}^{*}\right), \quad \tilde{w}_{0}=\operatorname{Re}\left(w_{0}^{*}\right),
$$

are the solutions of Eqs. (20a)-(20c). Equations (24a)(24c) suggest that the quasisteady solution takes the form

$$
\psi_{0}^{*}(r, \alpha, t)=\bar{\psi}_{0}(r, \alpha) e^{i t}, \quad w_{0}^{*}(r, \alpha, t)=\bar{w}_{0}(r, \alpha) e^{i t},
$$

where $\bar{\psi}_{0}$ and $\bar{w}_{0}$ are complex functions of $r$ and $\alpha$ in general. On substituting Eq. (26) into Eqs. (24a) $-(24 c)$, we obtain

$$
\begin{aligned}
& i \nabla^{2} \bar{\psi}_{0}-\frac{1}{R_{\Omega}} \nabla^{2} \nabla^{2} \bar{\psi}_{0}=-2 \Delta\left(\frac{\partial \bar{w}_{0}}{\partial r} \sin \alpha+\frac{1}{r} \frac{\partial \bar{w}_{0}}{\partial \alpha} \cos \alpha\right), \\
& i \bar{w}_{0}-\frac{1}{R_{\Omega}} \nabla^{2} \bar{w}_{0}=2 \Delta\left(\frac{1}{r} \frac{\partial \bar{\psi}_{0}}{\partial \alpha} \cos \alpha+\frac{\partial \bar{\psi}_{0}}{\partial r} \sin \alpha\right),
\end{aligned}
$$

with

$$
\bar{\psi}_{0}=\frac{\partial \bar{\psi}_{0}}{\partial r}=\bar{w}_{0}-(2 \Delta-1)=0 \quad \text { at } r=1 .
$$

On writing

$$
\bar{\psi}_{0}=\bar{\psi}_{0 r}+i \bar{\psi}_{0 i}, \quad \bar{w}_{0}=\bar{w}_{0 r}+i \bar{w}_{0 i},
$$

and using Eqs. (25) and (26), we have

$$
\tilde{\psi}_{0}=\bar{\psi}_{0 r} \cos t-\bar{\psi}_{0 i} \sin t, \quad \tilde{w}_{0}=\bar{w}_{0 r} \cos t-\bar{w}_{0 i} \sin t \text {. }
$$

Thus the source terms on the right-hand sides of Eqs. (21a) and (21b) can be written as

$$
\begin{array}{r}
\frac{1}{r}\left(\frac{\partial\left(\tilde{\psi}_{0}, \nabla^{2} \tilde{\psi}_{0}\right)}{\partial(r, \alpha)}+\frac{\partial\left(\psi_{R}, \nabla^{2} \tilde{\psi}_{0}\right)}{\partial(r, \alpha)}\right) \\
\quad=B_{0}+B_{1} \cos 2 t+B_{2} \sin 2 t,
\end{array}
$$

with

$$
\begin{aligned}
B_{0}= & \frac{1}{2 r}\left(\frac{\partial\left(\bar{\psi}_{0 r}, \nabla^{2} \bar{\psi}_{0 r}\right)}{\partial(r, \alpha)}+\frac{\partial\left(\bar{\psi}_{0 i}, \nabla^{2} \bar{\psi}_{0 i}\right)}{\partial(r, \alpha)}\right. \\
& \left.-\frac{\partial \nabla^{2} \bar{\psi}_{0 i}}{\partial \alpha} \sin \alpha+\frac{\partial \nabla^{2} \bar{\psi}_{0 i}}{\partial r} r \cos \alpha\right), \\
B_{1}= & \frac{1}{2 r}\left(\frac{\partial\left(\bar{\psi}_{0 r}, \nabla^{2} \bar{\psi}_{0 r}\right)}{\partial(r, \alpha)}-\frac{\partial\left(\bar{\psi}_{0 i}, \nabla^{2} \bar{\psi}_{0 i}\right)}{\partial(r, \alpha)}+\frac{\partial \nabla^{2} \bar{\psi}_{0 i}}{\partial \alpha} \sin \alpha\right. \\
& \left.-\frac{\partial \nabla^{2} \bar{\psi}_{0 i}}{\partial r} r \cos \alpha\right), \\
B_{2}= & \frac{1}{2 r}\left(\frac{\partial\left(\nabla^{2} \bar{\psi}_{0 r}, \bar{\psi}_{0 i}\right)}{\partial(r, \alpha)}+\frac{\partial\left(\nabla^{2} \bar{\psi}_{0 i}, \bar{\psi}_{0 r}\right)}{\partial(r, \alpha)}+\frac{\partial \nabla^{2} \bar{\psi}_{0 r}}{\partial \alpha} \sin \alpha\right. \\
& -\frac{\left.\partial \nabla^{2} \bar{\psi}_{0 r} r \cos \alpha\right),}{\partial r}
\end{aligned}
$$

and

$$
\frac{1}{r}\left(\frac{\partial\left(\tilde{\psi}_{0}, \tilde{w}_{0}\right)}{\partial(r, \alpha)}+\frac{\partial\left(\psi_{R}, \tilde{w}_{0}\right)}{\partial(r, \alpha)}\right)=C_{0}+C_{1} \cos 2 t+C_{2} \sin 2 t,
$$

with

$$
\begin{aligned}
C_{0}= & \frac{1}{2 r}\left(\frac{\partial\left(\bar{\psi}_{0 r}, \bar{w}_{0 r}\right)}{\partial(r, \alpha)}+\frac{\partial\left(\bar{\psi}_{0 i}, \bar{w}_{0 i}\right)}{\partial(r, \alpha)}-\frac{\partial \bar{w}_{0 i}}{\partial \alpha} \sin \alpha\right. \\
& \left.+\frac{\partial \bar{w}_{0 i}}{\partial r} r \cos \alpha\right), \\
C_{1}= & \frac{1}{2 r}\left(\frac{\partial\left(\bar{\psi}_{0 r}, \bar{w}_{0 r}\right)}{\partial(r, \alpha)}-\frac{\partial\left(\bar{\psi}_{0 i}, \bar{w}_{0 i}\right)}{\partial(r, \alpha)}+\frac{\partial \bar{w}_{0 i}}{\partial \alpha} \sin \alpha\right. \\
& \left.-\frac{\partial \bar{w}_{0 i}}{\partial r} r \cos \alpha\right), \\
C_{2}= & \frac{1}{2 r}\left(\frac{\partial\left(\bar{w}_{0 r}, \bar{\psi}_{0 i}\right)}{\partial(r, \alpha)}+\frac{\partial\left(\bar{w}_{0 i}, \bar{\psi}_{0 r}\right)}{\partial(r, \alpha)}+\frac{\partial \bar{w}_{0 r}}{\partial \alpha} \sin \alpha\right. \\
& \left.-\frac{\partial \bar{w}_{0 r}}{\partial r} r \cos \alpha\right) .
\end{aligned}
$$

These source terms suggest that the first-order components, $\psi_{1}$ and $w_{1}$, in Eqs. (21a)-(21c), can be expressed as

$$
\begin{aligned}
\tilde{\psi}_{1}(r, \alpha, t)= & \widetilde{\psi}_{1 S}(r, \alpha)+\operatorname{Re}\left[\bar{\psi}_{1 \alpha}(r, \alpha) e^{i 2 t}\right] \\
& +\operatorname{Im}\left[\bar{\psi}_{1 b}(r, \alpha) e^{i 2 t}\right] \\
\tilde{w}_{1}(r, \alpha, t)= & \widetilde{w}_{1 S}(r, \alpha)+\operatorname{Re}\left[\bar{w}_{1 a}(r, \alpha) e^{i 2 t}\right] \\
& +\operatorname{Im}\left[\bar{w}_{1 b}(r, \alpha) e^{i 2 t}\right] .
\end{aligned}
$$

The primary interest for the first-order result is that the pulsating driving mechanism [see $\left.\mathrm{Eq} .{ }_{.}(15 \mathrm{c})\right]$ can generate a steady stream (denoted by $\tilde{w}_{1 S}$ and $\widetilde{\psi}_{1 S}$ ) via the nonlinear 
convection terms. By using Eqs. (30a) and (30b) and (21a)-(21c), the governing equations for $\left(\widetilde{\psi}_{1 S}, \widetilde{w}_{1 S}\right)$ are

$$
\begin{aligned}
& -\frac{1}{R_{\Omega}} \nabla^{2} \nabla^{2} \widetilde{\psi}_{1 S}+2 \Delta\left(\frac{\partial \widetilde{w}_{1 S}}{\partial r} \sin \alpha+\frac{1}{r} \frac{\partial \widetilde{w}_{1 S}}{\partial \alpha} \cos \alpha\right)=B_{0}, \\
& -\frac{1}{R_{\Omega}} \nabla^{2} \widetilde{w}_{1 S}-2 \Delta\left(\frac{1}{r} \frac{\partial \widetilde{\psi}_{1 S}}{\partial \alpha} \cos \alpha+\frac{\partial \widetilde{\psi}_{1 S}}{\partial r} \sin \alpha\right)=C_{0},
\end{aligned}
$$

with

$$
\tilde{\psi}_{1 S}=\frac{\partial \widetilde{\psi}_{1 S}}{\partial r}=\widetilde{w}_{1 S}=0 \quad \text { at } r=1 .
$$

For small values of $A$, the dimensionless rate of energy dissipation per unit mass, $\Phi$, can be expressed as

$$
\Phi=\Phi_{0}+A \Phi_{1}+O\left(A^{2}\right),
$$

where

$$
\begin{aligned}
\Phi_{0}= & 2\left\{\left(\frac{\partial \widetilde{u}_{0}}{\partial r}\right)^{2}+\left[\frac{1}{r}\left(\frac{\partial \widetilde{v}_{0}}{\partial \alpha}+\widetilde{u}_{0}\right)\right]^{2}\right\}+\left(\frac{1}{r} \frac{\partial \widetilde{w}_{0}}{\partial \alpha}\right)^{2} \\
& +\left(\frac{\partial \widetilde{w}_{0}}{\partial r}\right)^{2}+\left[\frac{1}{r} \frac{\partial \widetilde{u}_{0}}{\partial \alpha}+r \frac{\partial}{\partial r}\left(\frac{\widetilde{v}_{0}}{r}\right)\right]^{2}
\end{aligned}
$$

and

$$
\begin{aligned}
\Phi_{1}= & 4\left\{\left(\frac{\partial \widetilde{u}_{0}}{\partial r} \frac{\partial \widetilde{u}_{1}}{\partial r}\right)+\left[\frac{1}{r}\left(\frac{\partial \widetilde{v}_{0}}{\partial \alpha}+\widetilde{u}_{0}\right)\right]\left[\frac{1}{r}\left(\frac{\partial \widetilde{v}_{1}}{\partial \alpha}+\widetilde{u}_{1}\right)\right]\right\} \\
& +2\left\{\left(\frac{1}{r} \frac{\partial \widetilde{w}_{0}}{\partial \alpha}\right)\left(\frac{1}{r} \frac{\partial \widetilde{w}_{1}}{\partial \alpha}\right)+\left(\frac{\partial \widetilde{w}_{0}}{\partial r}\right)\left(\frac{\partial \widetilde{w}_{1}}{\partial r}\right)+\left[\frac{1}{r} \frac{\partial \widetilde{u}_{0}}{\partial \alpha}\right.\right. \\
& \left.\left.+r \frac{\partial}{\partial r}\left(\frac{\widetilde{v}_{0}}{r}\right)\right]\left[\frac{1}{r} \frac{\partial \widetilde{u}_{1}}{\partial \alpha}+r \frac{\partial}{\partial r}\left(\frac{\widetilde{v}_{1}}{r}\right)\right]\right\}
\end{aligned}
$$

with

$$
\widetilde{u}_{i}=\frac{1}{r} \frac{\partial \widetilde{\psi}_{i}}{\partial \alpha}, \quad \widetilde{v}_{i}=-\frac{\partial \widetilde{\psi}_{i}}{\partial r} ; \quad i=0,1 .
$$

Consequently,

$$
g_{R}=g_{0}+A g_{1}+O\left(A^{2}\right),
$$

according to Eq. (17), where $g_{0}$ and $g_{1}$ are associated with $\Phi_{0}$ and $\Phi_{1}$, respectively. With Eqs. (25), (26), (30a), and (30b), each term on the right-hand side of Eq. (33b) can be written in the following form:

$$
\begin{aligned}
\Phi_{1}= & {\left[f^{\circ}(r, \alpha) \cos t+f^{1}(r, \alpha) \sin t\right]\left[f^{2}(r, \alpha)\right.} \\
& \left.+f^{3}(r, \alpha) \cos 2 t+f^{4}(r, \alpha) \sin 2 t\right] .
\end{aligned}
$$

where $f^{0}, f^{1}, f^{2}, f^{3}$, and $f^{4}$ are related to $\left(\dot{\tilde{u}_{0}}, \widetilde{v}_{0}, \widetilde{w}_{0}\right)$ and $\left(\widetilde{u}_{1}, \widetilde{v}_{1}, \widetilde{w}_{1}\right)$ together with their spatial derivatives. It follows that

$$
g_{1}=\frac{1}{\pi R_{\Omega}} \int_{0}^{2 \pi} \int_{0}^{2 \pi} \int_{0}^{1} \Phi_{1}\left(r, \alpha, t ; R_{\Omega} \Delta\right) r d r d \alpha d t=0 .
$$

Hence,

$$
g_{R}=g_{0}+O\left(A^{2}\right),
$$

which implies that $g_{R}$ is a weak function of $A$ for small values of $A$.

\section{ASYMPTOTIC SOLUTIONS}

Perturbation solutions are obtained in this section for the limiting cases $R_{\Omega}<1$ and $R_{\Omega} \geqslant 1$ with finite values of $\Delta$ and small values of $A$.

\section{A. Asymptotic solution for $R_{\Omega} \ll 1$}

Consider first the zeroth-order solution. For small $R_{\Omega}$, we may expand the solutions for Eqs. (27a) $-(27 \mathrm{c})$ by regular perturbation series as

$$
\begin{aligned}
\bar{\psi}_{0}(r, \alpha)= & \bar{\psi}_{00}(r, \alpha)+R_{\Omega} \bar{\psi}_{01}(r, \alpha)+R_{\Omega}^{2} \bar{\psi}_{02}(r, \alpha) \\
& +R_{\Omega}^{3} \bar{\psi}_{03}(r, \alpha)+\cdots, \\
\bar{w}_{0}(r, \alpha)= & \bar{w}_{00}(r, \alpha)+R_{\Omega} \bar{w}_{01}(r, \alpha)+R_{\Omega}^{2} \bar{w}_{02}(r, \alpha) \\
& +R_{\Omega}^{3} \bar{w}_{03}(r, \alpha)+\cdots .
\end{aligned}
$$

The governing equations for $\bar{\psi}_{00}, \bar{\psi}_{01}, \bar{\psi}_{02}, \ldots$ and $\bar{w}_{00}, \bar{w}_{01}$, $\bar{w}_{02}, \ldots$ are obtained by substituting Equs. (35a) and (35b) into Eqs. (27a) $-(27 c)$, and collecting the terms of equal powers of $R_{\Omega}$. The solutions up to the third order are $\bar{\psi}_{0}=\bar{\psi}_{0 r}+i \bar{\psi}_{0 i}$

$$
\begin{aligned}
= & \left(R_{\Omega}^{3} \frac{\Delta(2 \Delta-1)}{4608}\left(6 r-13 r^{3}+8 r^{5}-r^{7}\right) \sin \alpha\right) \\
& +i\left(R_{\Omega}^{2} \frac{\Delta(2 \Delta-1)}{192}\left(r-2 r^{3}+r^{5}\right) \sin \alpha\right),
\end{aligned}
$$

$$
\begin{aligned}
\bar{w}_{0}= & \bar{w}_{0 r}+i \bar{w}_{0 i} \\
= & {\left[(2 \Delta-1)\left(1+\frac{R_{\Omega}^{2}}{64}\left(-3+4 r^{2}-r^{4}\right)\right)\right]+i[(2 \Delta-1)} \\
& \times\left(\frac{R_{\Omega}}{4}\left(r^{2}-1\right)+\frac{R_{\Omega}^{3}}{2304}\left(19-27 r^{2}+9 r^{4}-r^{6}\right)\right. \\
& +\frac{R_{\Omega}^{3} \Delta^{2}}{2304}\left(2-6 r^{2}+6 r^{4}-2 r^{6}\right) \\
& \left.\left.+\frac{R_{\Omega}^{3}}{4608} \Delta^{2}\left(5 r^{2}-8 r^{4}+3 r^{6}\right) \cos 2 \alpha\right)\right] .
\end{aligned}
$$

It follows from Eq. (36a) that the maximum value of $\bar{\psi}_{0 i}$ equals $1.491 \times 10^{-3} \Delta(2 \Delta-1) R_{\Omega}^{2}$, which occurs at $r=0.4472, \alpha=90^{\circ}$, and the maximum value of $\bar{\psi}_{0 r}$ equals $3.606 \times 10^{-4}(2 \Delta-1) \Delta R_{\Omega}^{3}$, which occurs at $r=0.4352$, $\alpha=90^{\circ}$. When $R_{\Omega}=0.1$ and $\Delta=1$, the numerical results in Sec. $V$ for the maximum values of $\bar{\psi}_{0 i}$ and $\bar{\psi}_{0 r}$ together with their locations are $1.5 \times 10^{-5} \quad\left(r=0.45, \alpha=90^{\circ}\right)$ and $3.6 \times 10^{-7}\left(r=0.425, \alpha=90^{\circ}\right)$, respectively, which agree nicely with the asymptotic results.

It is interest to see the first-order steady term $\left(\widetilde{\psi}_{1 S}, \widetilde{w}_{1 S}\right)$ associated with the effect of finite values of $A$. The source terms on the right-hand sides of Eqs. (31a) and (31b) can be evaluated by using Eqs. (36a) and (36b) as 


$$
\begin{aligned}
& B_{0}=\frac{\Delta(2 \Delta-1)}{16} R_{\Omega}^{2} r^{2} \sin 2 \alpha+O\left(R_{\Omega}^{4}\right), \\
& C_{0}=\frac{(2 \Delta-1)}{4} R_{\Omega} r \cos \alpha+O\left(R_{\Omega}^{3}\right) .
\end{aligned}
$$

With these source terms, a simple scaling analysis of Eqs. (31a) and (31b) shows that $\widetilde{w}_{1 S \sim R} \sim R_{\Omega}^{2}$ and $\widetilde{\psi}_{1 S} \sim R_{\Omega}^{3}$. Thus the equations governing the leading-order terms, according to Eqs. (31a) and (31b), are

$$
\begin{aligned}
& -\frac{1}{R_{\Omega}} \nabla^{2} \nabla^{2} \widetilde{\psi}_{1 S}+2 \Delta\left(\frac{\partial \widetilde{w}_{1 S}}{\partial r} \sin \alpha+\frac{1}{r} \frac{\partial \tilde{w}_{1 S}}{\partial \alpha} \cos \alpha\right) \\
& =R_{\Omega}^{2} \frac{\Delta(2 \Delta-1)}{16} r^{2} \sin 2 \alpha, \\
& -\frac{1}{R_{\Omega}} \nabla^{2} \widetilde{w}_{1 S}=R_{\Omega} \frac{(2 \Delta-1)}{4} r \cos \alpha .
\end{aligned}
$$

Although the steady stream, $\widetilde{w}_{1 S}$ and $\widetilde{\psi}_{1 S}$, are generated by the source terms associated with the nonlinear convection effect, $\widetilde{w}_{1 S}$ and $\psi_{1 S}$ are coupled together through the Coriolis terms in general according to Eqs. (31a) and (31b). However, Eqs. (31a) and (31b) reduce to Eqs. (37a) and (37b) as $R_{\Omega} \rightarrow 0$, and thus $\widetilde{w}_{1 S}$ and $\widetilde{\psi}_{1 S}$ are decoupled from each other. The distribution of $\tilde{w}_{1 S}$ is mainly due to the source term, according to Eq. (37b), but the distribution of $\widetilde{\psi}_{1 s}$ depends on both the source term and the Coriolis term in Eq. (37a). The solution of Eqs. (37a) and (37b) subject to Eq. (31c) are

$$
\begin{aligned}
\tilde{\psi}_{1 S}= & \left(\frac{(2 \Delta-1) \Delta}{3072}\left(-r^{2}+2 r^{4}-r^{6}\right) \sin 2 \alpha\right) R_{\Omega}^{3} \\
& +O\left(R_{\Omega}^{5}\right), \\
\widetilde{w}_{1 S}= & \left(\frac{(2 \Delta-1)}{32}\left(r-r^{3}\right) \cos \alpha\right) R_{\Omega}^{2}+O\left(R_{\Omega}^{4}\right) .
\end{aligned}
$$

The associated velocity components of the steady secondary flow are

$$
\begin{aligned}
\tilde{u}_{1 S} & =\frac{1}{r} \frac{\partial \widetilde{\psi}_{1 S}}{\partial \alpha} \\
& =\left(\frac{(2 \Delta-1) \Delta}{1536}\left(-r+2 r^{3}-r^{5}\right) \cos 2 \alpha\right) R_{\Omega}^{3}+O\left(R_{\Omega}^{5}\right) \\
\widetilde{v}_{1 S} & =-\frac{\partial \widetilde{\psi}_{1 S}}{\partial r} \\
& =\left(\frac{(2 \Delta-1) \Delta}{1536}\left(r-4 r^{3}+3 r^{5}\right) \sin 2 \alpha\right) R_{\Omega}^{3}+O\left(R_{\Omega}^{5}\right)
\end{aligned}
$$

Equations (38a) $-(38 d)$ show that the steady stream is consisted of a counter flow along the axial direction and two pairs of symmetric counter-rotating vortices in the cross-sectional plane. Note that $\widetilde{w}_{1 S}$ is symmetric about the $x^{\prime}$ axis in Fig. 5 but antisymmetric about the $y^{\prime}$ axis, while $\widetilde{\psi}_{1 S}$ is antisymmetric about both the $x^{\prime}$ and $y^{\prime}$ axes, according to Eqs. (22b) and (23b). When $2 \Delta-1$ is positive, the axial flow is positive in the first and the fourth quadrants of Fig. 5, but is negative in the second and the third quadrants. Such axial flow direction is reversed if $2 \Delta-1$ is negative. The directions of the counter-rotating vorticies can be identified from the sign of $\widetilde{u}_{1 S}$ along $\alpha=0$ and $\alpha=\pi$ in Eq. (38c). Here, $\tilde{u}_{1 S}$ is negative along $\alpha=0$ but is positive along $\alpha=\pi / 2$ when $2 \Delta-1>0$, and vice versa when $2 \Delta$ $-1<0$.

The normalized energy dissipation per cycle according to Eqs. (17a) and (34b) for $R_{\Omega}<1$ is

$$
\begin{aligned}
g_{R 0}= & (2 \Delta-1)^{2} \pi\left[\frac{1}{8} R_{\Omega}-\left(\frac{11}{3072}+\frac{1}{4608} \Delta^{2}\right) R_{\Omega}^{3}\right. \\
& \left.+O\left(R_{\Omega}^{5}\right)\right]+O\left(A^{2}\right) .
\end{aligned}
$$

On the other hand, the result, according to the stationary pipe flow model in the limit $R_{\Omega} \rightarrow 0$, is

$$
g_{S 0}=\pi\left(\frac{R_{\Omega}}{8}-\frac{11}{3072} R_{\Omega}^{3}+O\left(R_{\Omega}^{5}\right)\right),
$$

which can be obtained by using Alfriend's result, or by solving the quasisteady solution of Eqs. (8a) and (8b) using regular perturbation expansion for small value of $R_{\Omega}$. Here the subscript " 0 " for both $g_{R}$ and $g_{S}$ is employed to refer that $g_{R}$ and $g_{S}$ are evaluated under the asymptotic condition $R_{\Omega} \rightarrow 0$. It follows from Eqs. (39a) and (39b) that

$$
\frac{g_{R 0}}{g_{s 0}}=(2 \Delta-1)^{2}\left(1-\frac{1}{576} \Delta^{2} R_{\Omega}^{2}+O\left(R_{\Omega}^{4}\right)\right)+O\left(A^{2}\right),
$$

which equals to unity when $\Delta=0$, decreases as $\Delta$ increases from zero, equals to zero when $\Delta=0.5$, and then increases approximately with $(2 \Delta-1)^{2}$ when $\Delta>0.5$ provided that $R_{\Omega}^{2} \Delta^{2} / 576$ is sufficiently less than unity.

\section{B. Asymptotic solution for $R_{\Omega} \gg 1$}

As $R_{\Omega}=O\left(10^{2}\right)-O\left(10^{3}\right)$ for the cases studied by Alfriend, ${ }^{3,8}$ it is of practical interest to study the asymptotic solution for large values of $\boldsymbol{R}_{\boldsymbol{\Omega}}$. When $\boldsymbol{R}_{\Omega} \geqslant 1$, we propose that most of the deformation is confined to a narrow region next to the pipe wall, and is called the boundary layer region. The rest of the flow field is called the core region, which is essentially inviscid. The thickness of the boundary layer $\delta_{B L}^{\prime}$ is of order $a R_{\Omega}^{-1 / 2}$ according to a scaling analysis of the equations of motion. The boundary layer and the core region are governed by simpler equations, which will be solved separately and matched in a common region to give a uniformly valid solution of the whole field. Consider the case when $A \rightarrow 0$ and $\Delta=O(1)$. As $\delta_{B L}^{\prime}$ is the correct length scale in the $r^{\prime}$ direction instead of $a$ for the fluid motion inside the boundary layer, Eqs. (27a) $-(27 c)$ had to be rescaled first before carrying out the analysis. Scaling analysis shows that $\bar{u}_{0}=\bar{\psi}_{0}=O\left(R_{\Omega}^{-1 / 2}\right), \bar{v}_{0}=O(1)$, and $\bar{w}_{0}=O(1)$ inside the 
boundary layer. On setting $\eta=(1-r) R_{\Omega}^{1 / 2}, \hat{w}_{0}=\bar{w}_{0}$, and $\hat{\psi}_{0}=\bar{\psi}_{0} R_{\Omega}^{1 / 2}$, we can rewrite Eqs. (27a) and (27b) in terms of the boundary layer coordinates $(\eta, \alpha)$ as follows:

$$
\begin{aligned}
i R_{\Omega}^{1 / 2} & \frac{\partial^{2} \hat{\psi}_{0}}{\partial \eta^{2}}-i \frac{\partial \hat{\psi}_{0}}{\partial \eta}-R_{\Omega}^{1 / 2} \frac{\partial^{4} \hat{\psi}_{0}}{\partial \eta^{4}}+2 \frac{\partial^{3} \hat{\psi}_{0}}{\partial \eta^{3}} \\
= & 2 \Delta R_{\Omega}^{1 / 2} \frac{\partial \hat{w}_{0}}{\partial \eta} \sin \alpha-2 \Delta \frac{\partial \hat{w}_{0}}{\partial \alpha} \cos \alpha+O\left(R_{\Omega}^{-1 / 2}\right), \\
i \hat{w}_{0} & -\frac{\partial^{2} \hat{w}_{0}}{\partial \eta^{2}}+R_{\Omega}^{-1 / 2} \frac{\partial \hat{w}_{0}}{\partial \eta} \\
& =2 \Delta R_{\Omega}^{-1 / 2} \frac{\partial \hat{\psi}_{0}}{\partial \alpha} \cos \alpha-2 \Delta \frac{\partial \hat{\psi}_{0}}{\partial \eta} \sin \alpha+O\left(R_{\Omega}^{-1}\right),
\end{aligned}
$$

which govern the flow inside the boundary layer. Equations (41a) and (41b) suggest

$$
\begin{aligned}
& \hat{\psi}_{0}(\eta, \alpha)=\hat{\psi}_{00}(\eta, \alpha)+R_{\Omega}^{-1 / 2} \hat{\psi}_{01}(\eta, \alpha)+\cdots, \\
& \hat{w}_{0}(\eta, \alpha)=\hat{w}_{00}(\eta, \alpha)+R_{\Omega}^{-1 / 2} \hat{w}_{01}(\eta, \alpha)+\cdots .
\end{aligned}
$$

The equations governing the leading-order terms of the fluid motion inside the boundary layer are

$$
\begin{aligned}
& i \frac{\partial^{2} \hat{\psi}_{00}}{\partial \eta^{2}}-\frac{\partial^{4} \hat{\psi}_{00}}{\partial \eta^{4}}=2 \Delta \frac{\partial \hat{w}_{00}}{\partial \eta} \sin \alpha \\
& i \hat{w}_{00}-\frac{\partial^{2} \hat{w}_{00}}{\partial \eta^{2}}=-2 \Delta \frac{\partial \hat{\psi}_{00}}{\partial \eta} \sin \alpha .
\end{aligned}
$$

These two equations are solved subjected to the nonslip condition at the wall,

$$
\hat{w}_{00}-(2 \Delta-1)=\hat{\psi}_{00}=\frac{\partial \hat{\psi}_{00}}{\partial \eta}=0 \text { at } \eta=0
$$

and the matching condition at the outer edge of the boundary layer. The scale for $\bar{\psi}$ in the core region is of order $R_{\Omega}^{-1 / 2}$ by considering the conservation of mass in the cross sectional plane $(r, \alpha)$ for the whole region, i.e., the boundary layer plus the core. As the length scale in the core is of order $a$, both $\bar{u}$ and $\bar{v}$ are also of order $R_{\Omega}^{-1 / 2}$ there. Since the flow is essentially inviscid in the core, $\bar{w}$ in the core is also of order $R_{\Omega}^{-1 / 2}$ by balancing the unsteady term to the Coriolis term. The velocity componcnts $\bar{w}$ and $\bar{v}$ inside the boundary layer are of order $R_{\Omega}^{1 / 2}$ greater than those inside the core region. The flow in the core region is thus essentially stagnant in comparing with the flow inside the boundary layer as $R_{\Omega} \rightarrow \infty$, which enables us to write the matching condition as

$$
\lim _{\eta \rightarrow \infty} \hat{w}_{00}(\eta, \alpha)=\lim _{\eta \rightarrow \infty} \frac{\partial \hat{\psi}_{00}}{\partial \eta}(\eta, \alpha)=0 .
$$

For a given value of $\alpha$, Eqs. (4.3a)-(43d) describe locally a Stokes' oscillating plate problem in a rotating frame or a "pulsating" Ekman layer flow. Equations (43a) and (43b) are ordinary differential equations regarding $\alpha$ as a parameter. The solutions of Eqs. (43a) -(43d) are, (i) for $\Delta<\frac{1}{2}$, and for $0 \leqslant \alpha<\alpha_{c}$ if $\Delta>\frac{1}{2}$,

$$
\begin{aligned}
\hat{w}_{00}= & \frac{(2 \Delta-1)}{2}\{\exp [-(1+i) b \eta] \\
& +\exp [-(1+i) q \eta]\} \\
\hat{v}_{00}= & \frac{\partial \hat{\psi}_{00}}{\partial \eta} \\
= & \frac{i(2 \Delta-1)}{2}\{\exp [-(1+i) b \eta] \\
& -\exp [-(1+i) q \eta]\} \\
\hat{\psi}_{00}= & \frac{(1+i)(2 \Delta-1)}{2 \sqrt{2}}\left(\frac{1}{b \sqrt{2}}\{1-\exp [-(1+i) b \eta]\}\right. \\
& \left.-\frac{1}{q \sqrt{2}}\{1-\exp [-(1+i) q \eta]\}\right)
\end{aligned}
$$

(ii) for $\alpha_{c}<\alpha \leqslant \pi / 2$ if $\Delta>\frac{1}{2}$,

$$
\begin{aligned}
\hat{w}_{00}= & \frac{(2 \Delta-1)}{2}\{\exp [-(1+i) b \eta] \\
& +\exp [-(1-i) q \eta]\}, \\
\hat{v}_{00}= & \frac{\partial \hat{\psi}_{00}}{\partial \eta} \\
= & \frac{i(2 \Delta-1)}{2}\{\exp [-(1+i) b \eta] \\
& -\exp [-(1-i) q \eta]\},
\end{aligned}
$$

$$
\begin{aligned}
\hat{\psi}_{00}= & \frac{(1+i)(2 \Delta-1)}{2 \sqrt{2}}\left(\frac{1}{b \sqrt{2}}\{1-\exp [-(1+i) b \eta]\}\right. \\
& \left.-\frac{i}{q \sqrt{2}}\{1-\exp [-(1-i) q \eta]\}\right)
\end{aligned}
$$

where

$$
\begin{aligned}
& b=[(1+2 \Delta \sin \alpha) / 2]^{1 / 2}, \\
& q=[(|1-2 \Delta \sin \alpha|) / 2]^{1 / 2},
\end{aligned}
$$

$\alpha_{c}=\sin ^{-1}[1 /(2 \Delta)]$, and $\hat{v}_{00}$ is the circumferential velocity component. For $R_{\Omega} \geqslant 1$, most of the deformation occurs inside the boundary layer and hence most of the energy is also dissipated there. The dimensionless rate of energy dissipation expressed in terms of the boundary layer coordinates $(\eta, \alpha)$ is

$$
\Phi=\left\{R_{\Omega}\left[\left(\frac{\partial \hat{w}_{0}}{\partial \eta}\right)^{2}+\left(-\frac{\partial^{2} \hat{\psi}_{0}}{\partial \eta^{2}}\right)^{2}\right]+O\left(R_{\Omega}^{1 / 2}\right)\right\}+O\left(A^{2}\right)
$$

The function $g_{R}$ defined in Eq. (17) can also be expressed in terms of the boundary layer coordinates:

$$
g_{R}=\frac{1}{\pi R_{\Omega}^{3 / 2}} \int_{0}^{2 \pi} \int_{0}^{2 \pi} \int_{0}^{\infty} \Phi(\eta, \alpha, t ; \Delta, A) d \eta d \alpha d t .
$$




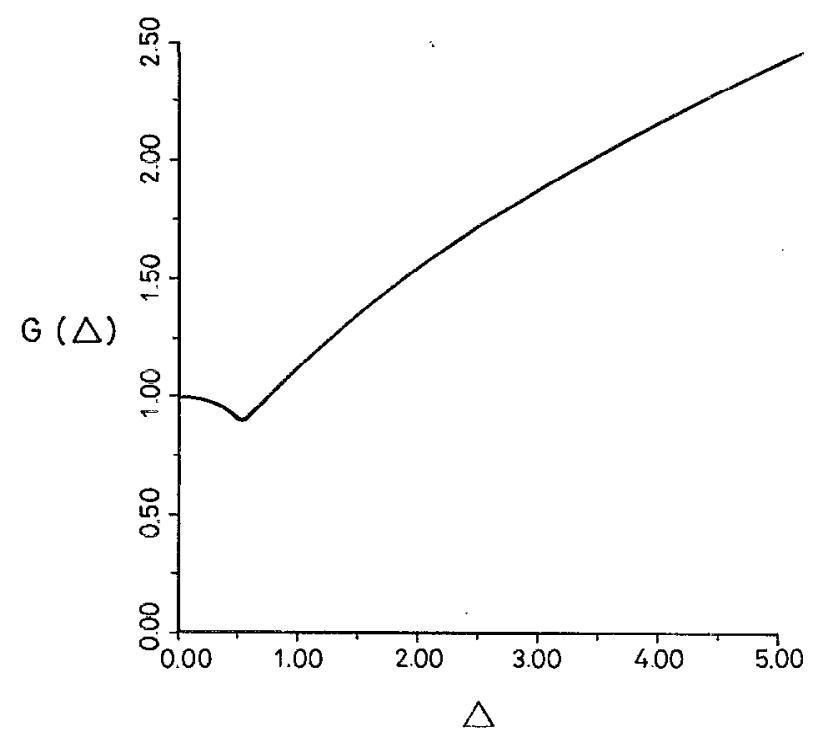

FIG. 6. Variation of the function $G(\Delta)$ for the asymptotic analysis when $R_{\Omega}>1$.

By using Eqs. (44), (45), (46), and (47), we obtain the function $g_{R}$ in the limit as $R_{\Omega} \rightarrow \infty$, and denote it by $g_{R \infty}$. The result is

$$
\frac{g_{R \infty}}{g_{S \infty}=}(2 \Delta-1)^{2} G(\Delta)+O\left(R_{\Omega}^{-1 / 2}\right)+O\left(A^{2}\right),
$$

where

$$
\begin{aligned}
G(\Delta)= & \frac{1}{\pi}\left(\int _ { 0 } ^ { \pi / 2 } \left[(1+2 \Delta \sin \alpha)^{1 / 2}\right.\right. \\
& \left.\left.+(|1-2 \Delta \sin \alpha|)^{1 / 2}\right] d \alpha\right)
\end{aligned}
$$

and

$$
g_{S_{\infty}}=\pi \sqrt{\frac{2}{R_{\Omega}}}+O\left(R_{\Omega}^{-1}\right) .
$$

Here, $g_{S \infty}$ denotes the function $g_{S}$ as $R_{\Omega} \rightarrow \infty$, according to the stationary pipe flow model. The function $G(\Delta)$ is calculated numerically and plotted in Fig. 6 . The rotation effect increases the energy dissipation substantially in the limiting case $R_{\Omega} \rightarrow \infty$ in general except when $\Delta<1$. Consider the cases studied by Alfriend ${ }^{3,8}$ and Hraster. ${ }^{7}$ The average experimental results for $g$ by Hraster are about 4.35 and 5.43 times Alfriend's theoretical results $\left(\approx g_{S}\right)$ for $\Delta=1.5$ and 2 , respectively (see Alfriend ${ }^{8}$ ). Here the rotating pipe flow model using Eq. (48) predicts $g_{R \infty} / g_{S \infty} \approx 5.36$ for $\Delta=1.5$ and 13.84 for $\Delta=2$, which agree much better with Hraster's experiments in comparison with Alfriend's theoretical prediction using the stationary pipe flow model.

For small $R_{\Omega}, g_{R 0}$ increases monotonically with $R_{\Omega}$, according to Eq. (39a). On the other hand, $g_{R}$ decreases monotonically as $R_{\Omega}$ increases for large $R_{\Omega}$ [see Eq. (48)]. Hence, we expect $g$ should attain a maximum at an intermediate value of $R_{\Omega}$ for a given value of $\Delta$, which is similar to the behavior of the stationary pipe flow results according to Bhuta and Koval. ${ }^{5}$ The numerical solution (see Sec. V) indeed shows this behavior and Eqs. (39a) and (48) form an envelope for the numerical result.

We have carried out the analysis for higher order solutions with an additional assumption that $\Delta<1$ (not shown in this paper). Further assumptions are required for cases with finite $\Delta$. We have not carried out such studies in the present work because of the following reasons: (1) Eq. $(48)$ is good enough for studying the energy dissipation for cases with large $R_{\Omega}$, (2) $R_{\Omega}=O(10)-O(100)$ for many practical situations, which can not fullfilled the asymptotic condition $R_{\Omega} \rightarrow \infty$, and (3) Eqs. (27a) $-(27 \mathrm{c}$ ) can be solved quite easily via numerical method in comparison with the theoretical analysis.

\section{NUMERICAL SOLUTION}

Detailed flow structure for $R_{\Omega}=O\left(10^{-1}\right)-O\left(10^{3}\right)$ and $\Delta=0-2$ have becn simulated numerically by solving Eqs. $(27 a)-(27 c)$ and (31a)-(31c). Consider first the zerothorder flow field. Equations (27a)-(27c) suggest that $\bar{w}_{0}$ is symmetric about the $x^{\prime}$ and $y^{\prime}$ axes, and $\bar{\psi}_{0}$ is symmetric about the $y^{\prime}$ axis but antisymmetric about the $x^{\prime}$ axis of Fig. 5 [see Eqs. (22a) and (23a)]. Hence, we need only to consider the first quadrant of the flow field for the calculations. By introducing the zeroth-order vorticity $\bar{\zeta}_{0}$,

$$
\bar{\zeta}_{0}=\nabla^{2} \bar{\psi}_{0},
$$

Eqs. (27a) and (27b) become

$$
i \bar{\zeta}_{0}-\frac{1}{R_{\Omega}} \nabla^{2} \bar{\xi}_{0}=-2 \Delta\left(\frac{\partial \bar{w}_{0}}{\partial r} \sin \alpha+\frac{1}{r} \frac{\partial \bar{w}_{0}}{\partial \alpha} \cos \alpha\right)
$$

and

$$
i \bar{w}_{0}-\frac{1}{R_{\Omega}} \nabla^{2} \bar{w}_{0}=2 \Delta\left(\frac{1}{r} \frac{\partial \bar{\psi}_{0}}{\partial \alpha} \cos \alpha+\frac{\partial \psi_{0}}{\partial r} \sin \alpha\right) .
$$

The boundary conditions for Eqs. (50a) $-(50 c)$ are

$$
\begin{aligned}
& \bar{\psi}_{0}(r, 0)=\frac{\partial \bar{\psi}_{0}}{\partial \alpha}\left(r, \frac{\pi}{2}\right)=\bar{\psi}_{0}(1, \alpha)=0, \\
& \bar{\zeta}_{0}(r, 0)=\frac{\partial \bar{\zeta}_{0}}{\partial \alpha}\left(r, \frac{\pi}{2}\right)=\bar{\zeta}_{0}(1, \alpha)-\frac{\partial^{2} \bar{\psi}_{0}}{\partial r^{2}}(1, \alpha)=0,
\end{aligned}
$$

and

$$
\frac{\partial \bar{w}_{0}}{\partial \alpha}(r, 0)=\frac{\partial \bar{w}_{0}}{\partial \alpha}\left(r, \frac{\pi}{2}\right)=\bar{w}_{0}(1, \alpha)-2 \Delta+1=0 .
$$

All the differential terms in Eqs. (50a) - (50c) and (51a)(51c) are replaced by central difference approximations, and the resulting finite difference equations constitute a complex linear algebraic system. The standard $L U$ decomposition method for inverting a matrix is employed to solve such linear complex system of algebraic equations. It requires $83 \mathrm{CPU}$ seconds in the CONVEX $\mathrm{C} 1$ computer to solve a case with 41 nonuniformly spaced grids in the $r$ direction and 19 uniformly spaced grids in the $\alpha$ direction. Details of the calculation procedures and finite difference equations can be found from $\mathrm{Ho}^{13}$ 


\section{A. Grid dependence}

Asymptotic analysis for $R_{\Omega} \geqslant 1$ in Sec. IV shows that the velocity gradient is large in the $r$ direction near the wall but is small in the $\alpha$ direction. Hence, we put $N$ uniformly spaced grids in the $\alpha$ direction, but employed $M$ grids in the $r$ direction with $M_{1}$ uniformly spaced grids in the core region and $M-M_{1}$ nonuniformly spaced grids in the region near the wall. Let $\bar{h}$ be the grid spacing between the uniformly spaced grids in the $r$ direction. Also let $\sigma$ be a factor less than unity, which is employed to specify the rate of change of the grid spacing between the nonuniformly spaced grids. In the following equation, $M_{1}, \bar{h}$, and $\sigma$ are related by

$$
\left(M_{1}-1\right) \bar{h}+\sum_{i=1}^{M-M_{1}} \sigma^{i} \bar{h}=1,
$$

which says that the grid spacing decreases from $\bar{h}$ continuously toward the wall at $r=1$, with the smallest grid spacing $\left(h_{\min }\right)$ next to the wall. Once $M, M_{1}$, and $\sigma$ are specified, $\bar{h}$ can be calculated directly from Eq. (52a) as

$$
\bar{h}=\left(M_{1}-1+\frac{\sigma}{1-\sigma}\left(1-\sigma^{\left(M-M_{1}\right)}\right)\right)^{-1} .
$$

For the present calculations, we employed the following grid systems: (1) grid \#1: $M=41, M_{1}=41, \sigma=0$, and $N=19$ (uniform grids), (2) grid $\# 2: M=41, M_{1}=31$, $\sigma=0.9$, and $N=19$, (3) grid \#3: $M=41, M_{1}=31, \sigma=0.8$, and $N=19$, (4) grid \#4: $M=41, M_{1}=31, \sigma=0.7$, and $N=19$. The grid spacing in the $\alpha$ direction for all these four grid systems is $5^{\circ}$. According to Eqs. (52a) and (52b), $\bar{h}=0.025, \quad 0.027885, \quad 0.029788$, and 0.030991 , and $h_{\min }=0.025,0.00972,0.0034$, and 0.00088 for grid \#1, $\# 2$, \#3, and \#4, respectively. The variation of $h$ is small but that of $h_{\min }$ is large for different grids.

Table I shows the normalized energy dissipation function $g$ for different values of $R_{\Omega}$ and $\Delta$ at $A=0$. It is found that smaller $h_{\min }$ is required for cases with larger values of $R_{\Omega}$. By requiring that the variation of $g$ be less than $1 \%$, we employ grid \#4 for cases when $R_{\Omega}>1000$, grid \#3 for cases when $R_{\Omega}=400-700$, grid \#2 for cases when $R_{\Omega}=100-200$, and grid $\# 1$ for cases when $R_{\Omega}<100$. Grid dependence test has also been carried out for different values of $N$ (i.e., the grid spacing in the $\alpha$ direction), and it is found that $N=19$ is accurate enough for the simulations.

\section{B. Validity of the computer program}

The computer program for generating the numerical results is checked by comparing with Hraster's experimental data for the energy dissipation and with the asymptotic solutions in Sec. IV for the detailed flow field. First we check against the energy dissipation. Figure 7 shows the numerical calculations for the normalized energy dissipation function $g$ (include $g=g_{R}$ for $\Delta \neq 0$ and $g=g_{S}$ for $\Delta=0$ ) together with the asymptotic solutions in Sec. IV. Also presented in Fig. 7 are the theoretical results based on the stationary pipe flow model (Bhuta and $\mathrm{Koval}^{5}$ and Alfriend ${ }^{3}$ ) and Hraster's ${ }^{7}$ experimental data. The numerical results agree nicely with the asymptotic solutions for
TABLE I. The normalized energy dissipation function $g$ for different grid systems at different values of $R_{\Omega}$ and $\Delta$.

\begin{tabular}{cccccc}
\hline$R_{\Omega}$ & $\Delta$ & Grid \#1 & Grid \#2 & Grid \#3 & Grid \#4 \\
\hline 1000 & 2.0 & 1.9703 & 1.9914 & 2.0147 & 2.0253 \\
1000 & 1.5 & 0.7589 & 0.7759 & 0.7844 & 0.7880 \\
1000 & 1.0 & 0.1544 & 0.1604 & 0.1621 & 0.1628 \\
1000 & 0.2 & 0.0451 & 0.0485 & 0.0491 & 0.0494 \\
1000 & 0.0 & 0.1268 & 0.1359 & 0.1376 & 0.1386 \\
400 & 2.0 & 3.0696 & 3.1277 & 3.1493 & 3.1580 \\
400 & 1.5 & 1.2050 & 1.2290 & 1.2363 & 1.2390 \\
400 & 1.0 & 0.2504 & 0.2560 & 0.2573 & 0.2581 \\
400 & 0.2 & 0.0740 & 0.0762 & 0.0767 & 0.0769 \\
400 & 0.0 & 0.2074 & 0.2134 & 0.2149 & 0.2155 \\
100 & 2.0 & 5.8727 & 5.9404 & 5.9538 & \\
100 & 1.5 & 2.3571 & 2.3802 & 2.3855 & \\
100 & 1.0 & 0.5073 & 0.5116 & 0.5128 & \\
100 & 0.2 & 0.1465 & 0.1476 & 0.1478 & \\
100 & 0.0 & 0.4089 & 0.4121 & 0.4125 & \\
10 & 2.0 & 11.983 & 12.017 & & \\
10 & 1.5 & 5.1914 & 5.2023 & & \\
10 & 1.0 & 1.2294 & 1.2310 & & \\
10 & 0.2 & 0.3975 & 0.3977 & & \\
10 & 0.0 & 1.0966 & 1.0972 & & \\
\hline \hline
\end{tabular}

$R_{\Omega} \ll 1$ when $R_{\Omega} \leqslant 1$, and with the asymptotic solutions for $R_{\Omega} \gg 1$ when $R_{\Omega}>50$. For $\Delta=0$, the present numerical calculations also agree with the results based on the stationary pipe flow model for all values of $R_{\Omega}$ except when $R_{\Omega} \leqslant 1$, which is due to the transient term in Alfriend's result. Note that only the quasisteady solution is analyzed in the

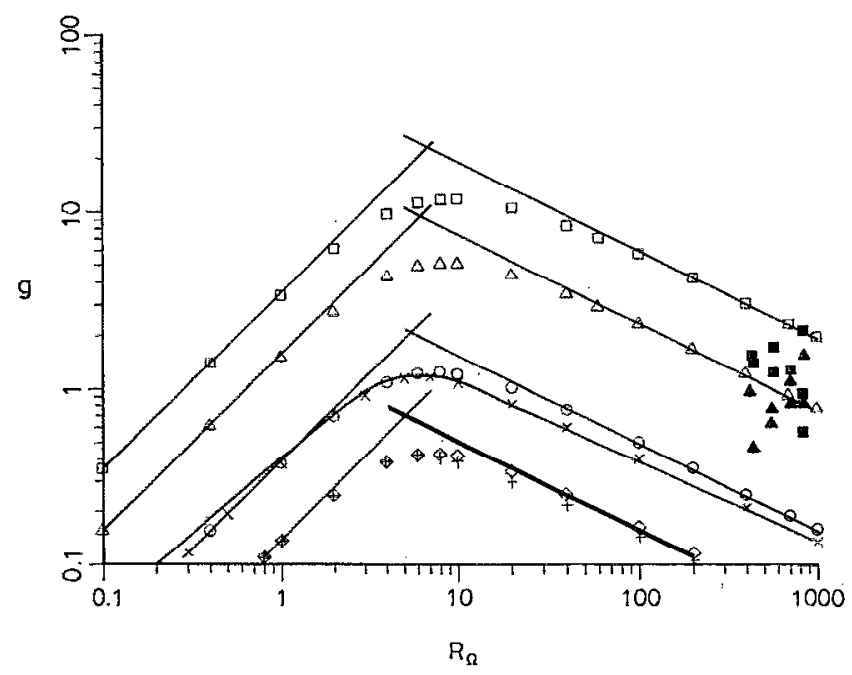

FIG. 7. Variation of the normalized energy dissipation function $g$ for different values of $R_{\Omega}$ and $\Delta$ when $A=0$. The curve shows the stationary pipe flow result by Bhuta and Koval. The straight lines with positive slopes, starting from top, are the asymptotic solutions for $R_{\Omega}<1$ when $\Delta=2,1.5,1,0.8$, and 0.2 , respectively (the last two lines coincide to each other). The straight lines with negative slopes, starting from top, are the asymptotic solutions for $R_{\Omega}>1$ when $\Delta=2,1.5,1,0.8$, and 0.2 , respectively. The numerical results are denoted by data points: " $\square$ " for $\Delta=2$, " $\Delta$ " for $\Delta=1.5$, "O" for $\Delta=1$, " $\times$ " for $\Delta=0$, " $\nabla$ " for $\Delta=0.8$, and " + " for $\Delta=0.2$. The solid squares and triangles represent the experimental results by Hraster when $\Delta=2$ and 1.5 , respectively. 


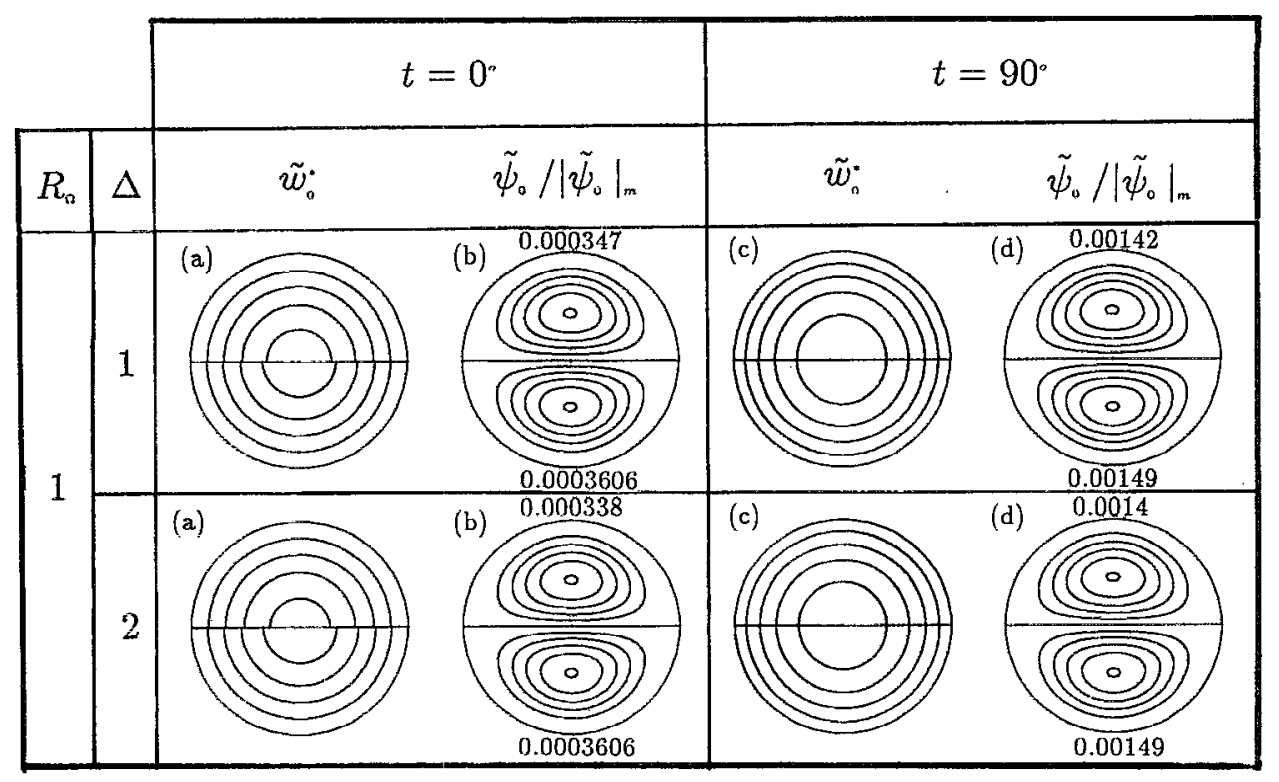

FIG. 8. Comparison between the numerical calculation (upper semicircle of each figure) and the asymptotic solution as $R_{\mathrm{\Omega}}<1$ (lower semicircle) for the zeroth-order results, $\tilde{w}_{0}^{*}$ and $\bar{\psi}_{0} /\left|\bar{\psi}_{0}\right|_{m}$, where $\left|\widetilde{\psi}_{0}\right|_{m}$ is the maximum value of $\left|\widetilde{\psi}_{0}\right|$. The contour values, starting from the boundary, are (a) 1.00 , $0.99,0.98,0.97,0.96$, (b) $0,0.2,0.4,0.6,0.8,0.99$, (c) $0,0.05,0.10,0.15,0.20$, and (d) $0,-0.2,-0.4,-0.6,-0.8,-0.99$. The value next to each semicircular figure of the $\widetilde{\psi}_{0} /\left|\widetilde{\psi}_{0}\right|_{m}$ contours is the value of $\left|\widetilde{\psi}_{0}\right|_{m}$ for that case.

present study, and the transient contribution to $g$ is larger for smaller $R_{\Omega}$ (see Alfriend ${ }^{3}$ ). The numerical results also indicate that there exists a maximum value of $g$ at $R_{\mathbf{\Omega}} \approx 10$ for a given $\Delta$. The numerical results for $\Delta=0.8$ and 1 coincide with those for $\Delta=0.2$ and 0 , respectively, for small values of $R_{\Omega}$, which can be expected from Eq. (39a). As suggested by the asymptotic results, the numerical solutions also indicate that $g\left(=g_{R}\right)=0$ for $\Delta=0.5, g_{R}<g_{S}$ for $\Delta$ approximately less than unity, and $g_{R} / g_{S}$ increases rapidly with $\Delta$ for $\Delta>1$. By comparing with Hraster's experiments, both the asymptotic and the numerical solutions in the present rotating pipe flow model agree nicely with the experiments for $\Delta=1.5$, but is about two times greater than those experimental results for $\Delta=2$. Recall that the experimental results are about five times greater than the theoretical prediction based on the stationary pipe flow model, $g_{S}$ (see Alfriend ${ }^{8}$ ).

The normalized flow properties defined as

$$
\left(\tilde{w}_{0}^{*}, \hat{w}_{0}^{*}, \hat{v}_{0}^{*}\right)=\frac{1}{2 \Delta-1}\left(\widetilde{w}_{0}, \hat{w}_{0}, \hat{v}_{0}\right)
$$

will be employed to express the following numerical results. Figure 8 compares the numerical solutions for $R_{\mathbf{\Omega}}=1$ with the asymptotic solutions for $R_{\Omega}<1$. Both the contours for $\widetilde{w}_{0}^{*}$ and $\widetilde{\psi}_{0} /\left|\widetilde{\psi}_{0}\right|_{m}$ are plotted in Fig. 8. Here $\left|\widetilde{\psi}_{0}\right|_{m}$ is the maximum value of $\left|\bar{\psi}_{0}\right|$. The numerical solutions (even $R_{\Omega}=1$ here) agree nicely with the asymptotic solutions for $R_{\Omega}<1$. The agreement is better for smaller value of $R_{\Omega}$. Figure 9 compares the numerical solutions with the asymptotic solutions for $R_{\Omega} \geqslant 1$ according to Eqs. (42a) and (42b), (44a) $-(44 \mathrm{c})$ and (45a) $-(45 \mathrm{c})$ at $\alpha=0^{\circ}$ and $90^{\circ}$ (see Fig. 5) when $R_{\Omega}=1000$. For $\Delta=0$, the flow is axisymmetric and the numerical solution agrees perfectly with the asymptotic result for all the time, $t$. The agreement between the numerical and the asymptotic solutions are also nice for $\Delta \neq 0$. The numerical solution for $R_{\Omega}=400$ (not shown here) also agrees fairly with the asymptotic solution.

\section{The zeroth-order flow field}

As the flow studied in this paper is quasisteady, $\widetilde{w}_{0}$ and $\widetilde{\psi}_{0}$ are periodic in $t$ with period $2 \pi$. Further Eqs. (28) show that

$$
\begin{aligned}
& \widetilde{w}_{0}(r, \alpha, t)=-\widetilde{w}_{0}(r, \alpha, \pi+t), \\
& \widetilde{\psi}_{0}(r, \alpha, t)=-\widetilde{\psi}_{0}(r, \alpha, \pi+t) .
\end{aligned}
$$

Thus the results for $t=0-\pi$ are sufficient to provide enough informations for the flow field. In this paper, we present the result at six distinct time instants; $t=0^{\circ}, 30^{\circ}$, $60^{\circ}, 90^{\circ}, 120^{\circ}$, and $150^{\circ}$, to show the time development of the flow field. The results at $t=180^{\circ}$ are the same as those at $t=0^{\circ}$ except the signs are changed. Many cases have been simulated and some of the typical cases are reported here. Figures 10-12 show the contours for the normalized axial velocity $\widetilde{w}_{0}^{*}$ and the normalized secondary streamfunction $\widetilde{\psi}_{0} /\left|\widetilde{\psi}_{0}\right|_{m}$ for different $\Delta$ 's at $R_{\Omega}=10,100$, and 1000 , respectively. Since the axial (primary) and the secondary flow are symmetric and antisymmetric, respectively, with respect to the $x^{\prime}$ axis in Fig. 5 [see Eq. (22a)], we plot $\widetilde{w}_{0}^{*}$ in the upper semicircle $(r=0-1, \alpha=0-\pi)$ and $\widetilde{\psi}_{0} /\left|\psi_{0}\right|_{m}$ in the lower semicircle $(r=0-1, \alpha=\pi$ $-2 \pi$ ) together in order to provide a more clear picture to illustrate the interaction between the primary and the secondary flow. The $\widetilde{\psi}_{0} /\left|\widetilde{\psi}_{0}\right|_{m}$ contours in the upper semicir- 


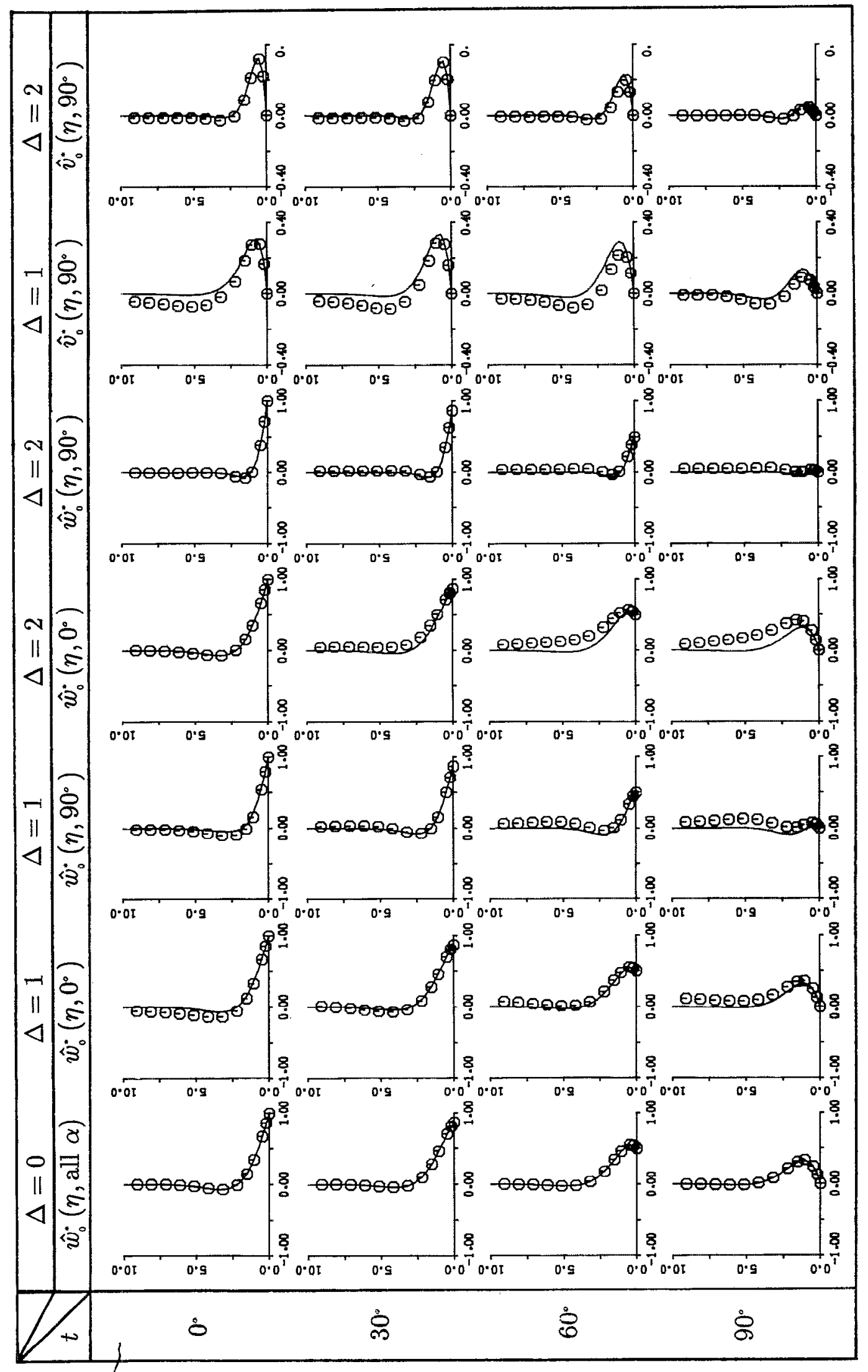

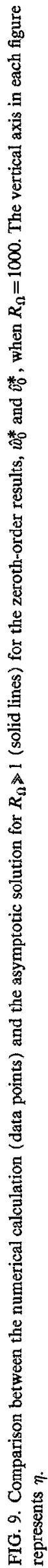




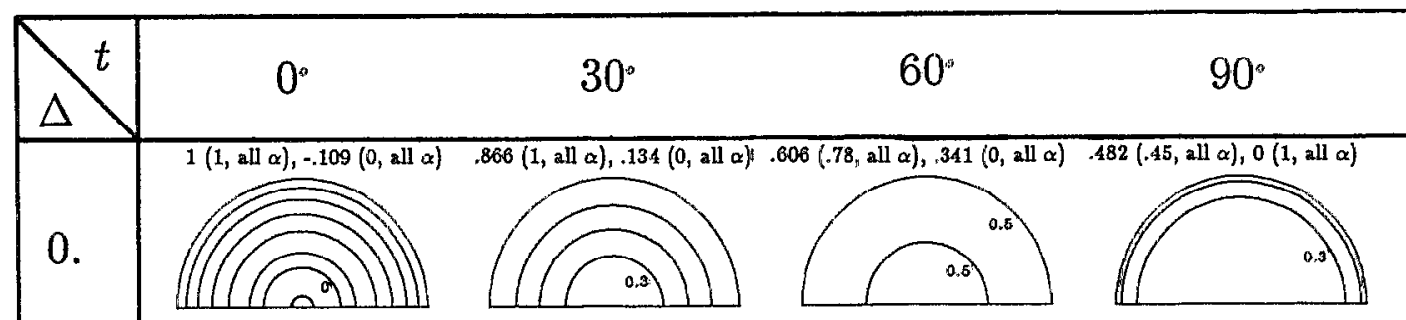

$120^{\circ}$

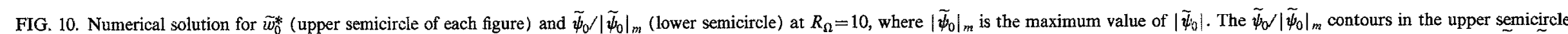

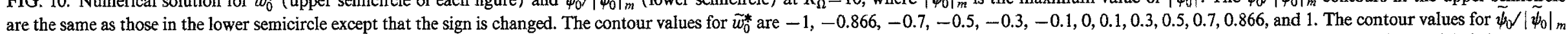

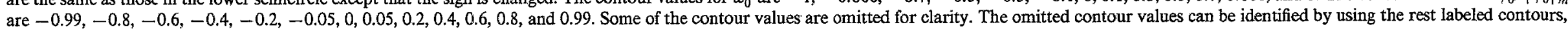

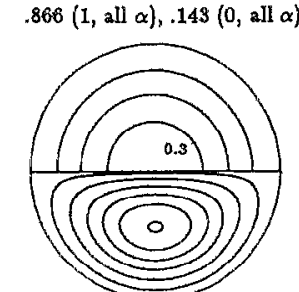

$.0073\left(.45,90^{\circ}\right), 0(1$, all $\alpha)$ $866(1$, all $\alpha), .318(0$, all $\alpha)$
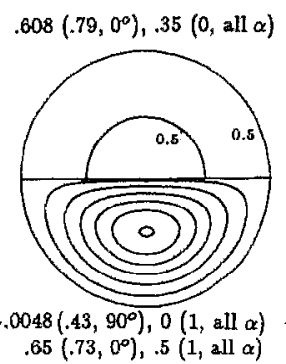

$1(1$, all $\alpha), 041(0$, all $\alpha)$

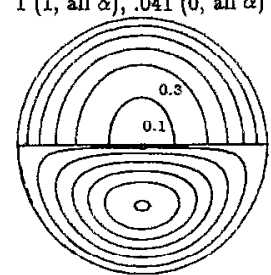

$.052\left(.48,90^{\circ}\right), 0(1$, all $\alpha)$ $1(1$, anl $\alpha), .157\left(.33,90^{\circ}\right)$

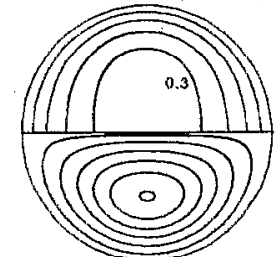

$.117\left(.5,90^{\circ}\right), 0(1$, all $\alpha)$ $1(I$, all $\alpha), .218\left(.45,90^{\circ}\right)$

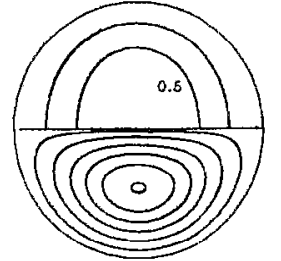

$.042\left(.45,90^{\circ}\right), 0(1$, all $\alpha)$ $.66(1$, all $\alpha), .424\left(.38,00^{\circ}\right)$

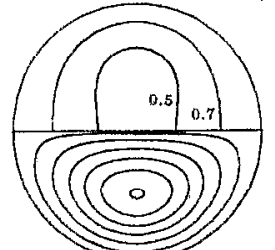

$084\left(48,90^{\circ}\right), 0(1$, all $\alpha)$

$.866(1$, all $\alpha), .479\left(.5,90^{\circ}\right)$

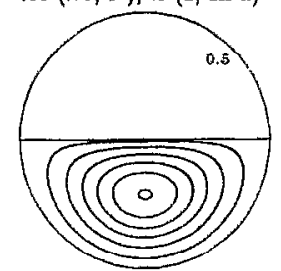

$.021\left(.43,90^{\circ}\right), 0(1$, all $\alpha)$

$.487\left(.42,0^{\circ}\right), 0(1$, all $\alpha)$

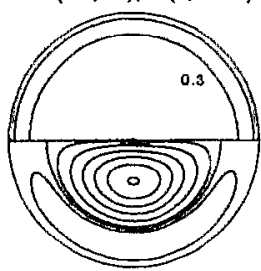

(2) $.568\left(.25,0^{\circ}\right), 0(1$, all $\alpha)$

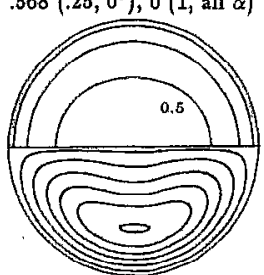

$\langle 1$, all $\alpha),-.0072\left(.63,90^{\circ}\right)$ $.599\left(.75,0^{\circ}\right), 0(1$, all $\alpha)$

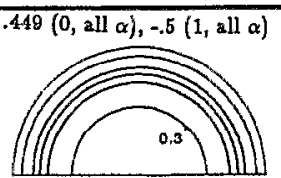

$.453(0$, all $\alpha),-.5(1$, all $\alpha)$

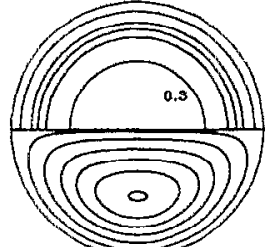

0 (1, all a) $0032\left(.53,90^{\circ}\right)$ $.47(0$, all $\alpha),-.5(1$, all $\alpha)$

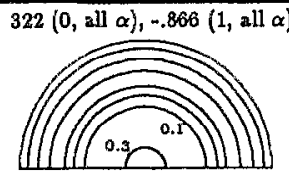

$.32(0$, all $\alpha),-.866(1$, all $\alpha)$

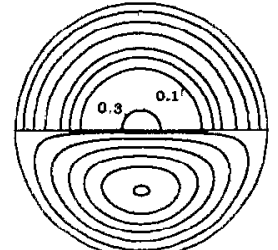

$0(1$, all $\alpha),-.0063\left(.48,90^{\circ}\right)$ $.248(0$, all $\alpha),-.866(1$, all $\alpha)$

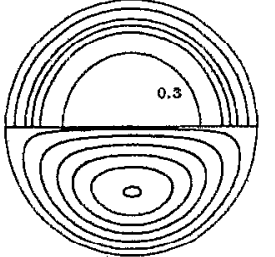

$0(1$, all $\alpha),-.0314\left(.5,90^{\circ}\right)$ $.434(0, \text { all } \alpha)_{1},-5(1$, all $\alpha)$

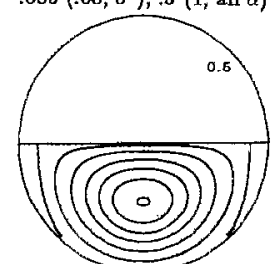

$.0285\left(.45,90^{\circ}\right),-00012(.93,2$
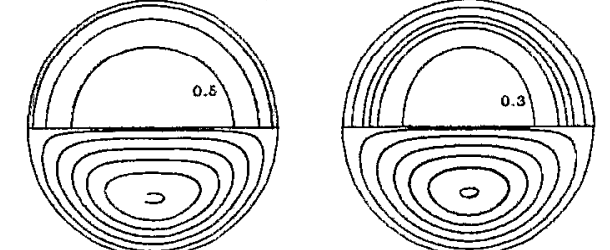

$.156\left(.25,90^{\circ}\right),-.866(1$, all $\alpha)$

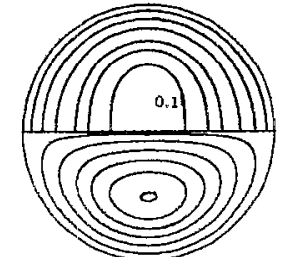

$0(1$, all $\alpha), \ldots .351(.55$,

$0(1$, all $\alpha),-.0882\left(.53,90^{\circ}\right)$

$(1$, all $\alpha),-.1179\left(.5,90^{\circ}\right)$
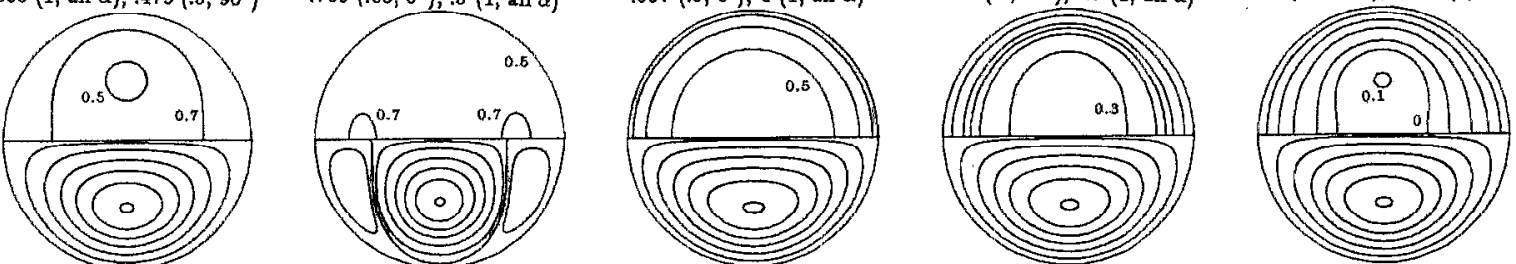

$\Delta=0$ 


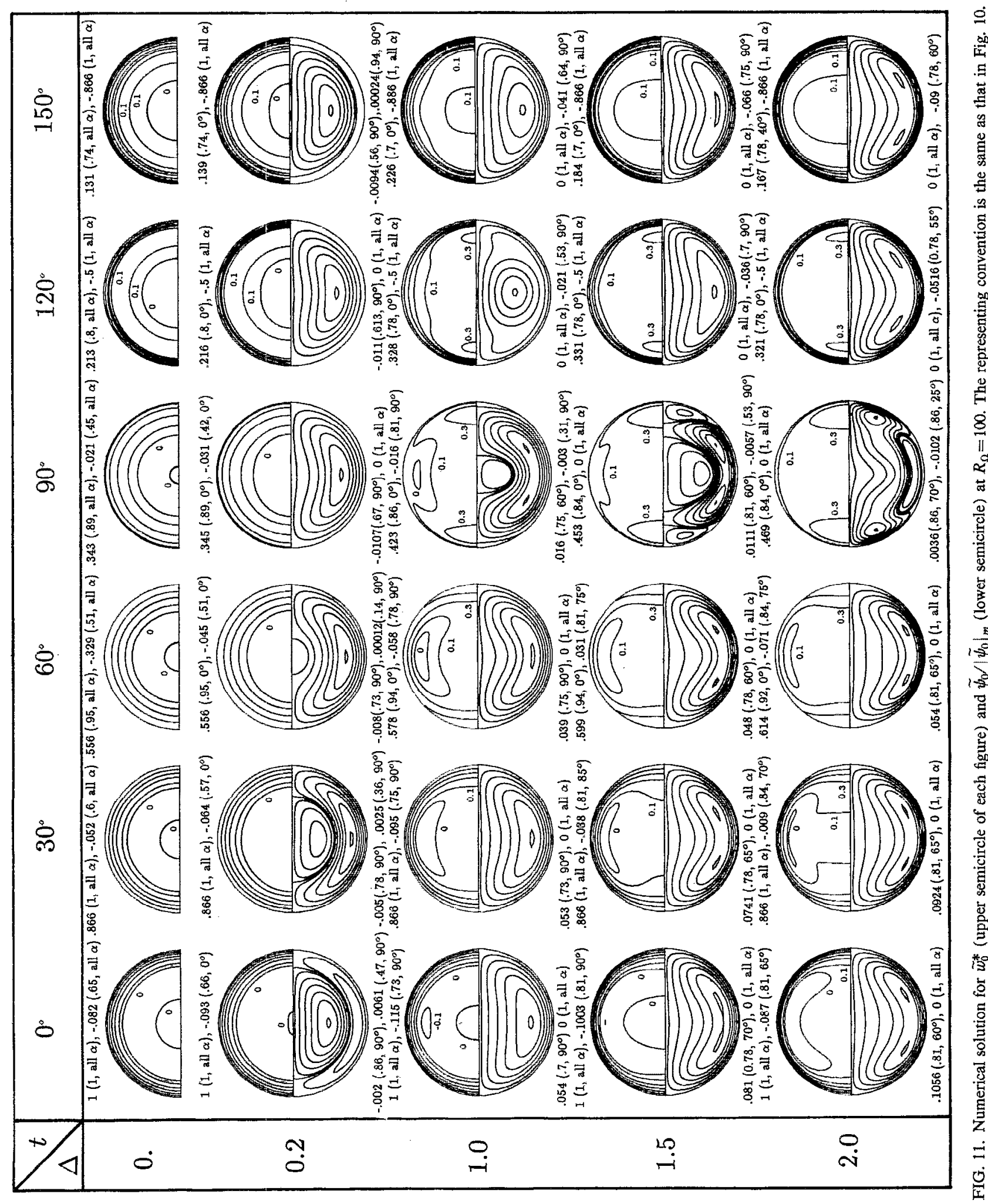




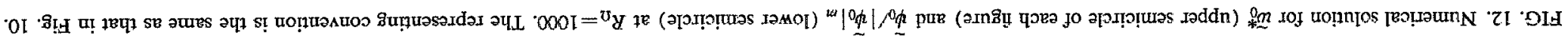

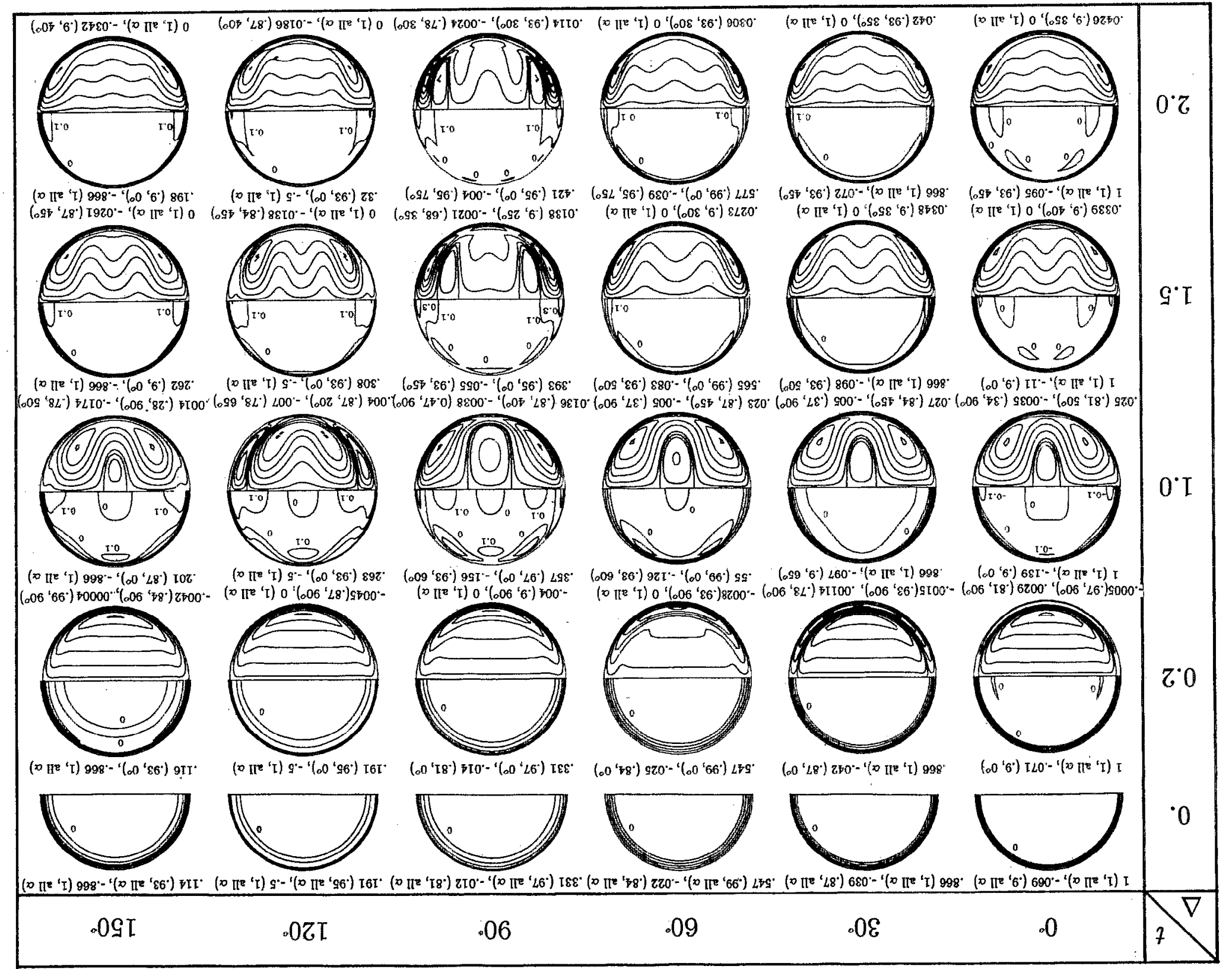


cle are the same as those in the lower semicircle except the sign is changed. At the pipe wall, $\tilde{w}_{0}^{*}=1,0.866,0.5,0$, -0.5 and -0.866 when $t=0,30^{\circ}, 60^{\circ}, 90^{\circ}, 120^{\circ}$, and $150^{\circ}$, respectively. Here, $\widetilde{w}_{0}^{*}$ varies from 1 to -1 during one cycle of the pipe wall oscillation. Thus we plot the normalized axial velocity contours when $\widetilde{w}_{0}^{*}=1,0.866,0.7,0.5$, $0.3,0.1,0,-0.1,-0.3,-0.5,-0.7,-0.866$, and -1 , and listed the maximum and minimum values of $\tilde{w}_{0}^{*}$ together with their locations in the upper $(r, \alpha)$ plane next to each semicircular areas of the $\widetilde{w}_{0}^{*}$ contours in Figs. 10-12. Some of the values of the contours are omitted for clarity, which can be inferred from the values and locations of the maximum and minimum $\widetilde{w}_{0}^{*}$ and the rest labeled contours. The strength and the direction of the secondary flow are characterized by the maximum (positive or zero)' and minimum (negative or zero) values of $\widetilde{\psi}_{0}$, which are also listed together with their locations in the first quadrant next to the $\widetilde{\psi}_{0} /\left|\widetilde{\psi}_{0}\right|_{m}$ contours in Figs. $10-12$. The contour values of $\psi_{0} /\left|\psi_{0}\right|_{m}$ are $0.99,0.8,0.6,0.4,0.2,0.05,0,-0.05$, $-0.2,-0.4,-0.6,-0.8$, and -0.99 , which are all omitted for clarity in Figs. 10-12. The value of a specified secondary stream function contours can be identified rather easily by using the values and the locations of the maximum and minimum $\widetilde{\psi}_{0}$, and keeping in mind that $\widetilde{\psi}_{0}=0$ at $r=1, \alpha=0$ and $\pi$. According to the maximum values of $\left|\widetilde{\psi}_{0}\right|_{m}$ and their location listed in Figs. 10-12, we found that the strength of the secondary flow incrcases as $\Delta$ increases for a given $\boldsymbol{R}_{\Omega}$. Although $\left|\bar{\psi}_{0}\right|_{m}$ decreases as $\boldsymbol{R}_{\boldsymbol{\Omega}}$ increases for a given $\Delta$, the locations of $\left|\widetilde{\psi}_{0}\right|_{m}$ vary significantly for different $R_{\Omega}$, and thus it is not obvious to tell whether the secondary velocity is increased or decreased as $R_{\Omega}$ increases. In all the axial velocity and secondary stream-function contours in Figs. $10-12$, the patterns are symmetric about $\alpha=\pi / 2$ or $3 \pi / 2$ (i.e., the $y^{\prime}$ axis in Fig. 5 ), according to Eq. (23a).

For $\Delta=0$, there is no secondary flow, the flow is axisymmetric and the contours for $\widetilde{w}_{0}^{*}$ are semicircular as shown by the figures on the first row of Figs. 10-12. For small $R_{\Omega}$ (say, $R_{\Omega}=10$ in Fig. 1 ), the viscous effect is important for most of the flow region and the whole body of fluid from $r=1$ to 0 is affected by the motion of the wall. However, when $R_{\Omega}=1000$, the viscous effect is confined in a thin region (the boundary layer) next to the wall, and a large amount of the flow region (the core) remains essentially stagnant. Note that there is an overshoot of the radial distribution of the axial velocity at the outer edge of the boundary layer (see the upper left figure of Fig. 9). When $\Delta \neq 0$, the secondary flow is generated by the Coriolis effect, which modifies the primary flow through the Coriolis term on the right-hand side of Eq. (27b), and alters the axial velocity profile from axisymmetric state. Such modified primary flow also affects the secondary flow, according to Eq. (27a), and hence the primary and the secondary flow are coupled to each other. For $\Delta=0.2$, the strength of the secondary flow is too weak (see the values of $\left|\psi_{0}\right|_{m}$ ) such that the axial velocity contours shown in Figs. 10-12 are more or less axisymmetric. The strength of the secondary flow becomes significant when $\Delta \geqslant 1$, and the $\widetilde{w}_{0}^{*}$ contours become not axisymmetric and complicated. The con- tours for $\widetilde{w}_{0}^{*}\left[=\tilde{w}_{0} /(2 \Delta-1)=\operatorname{Re}\left\{\bar{w}_{0} e^{i t}\right\} /(2 \Delta-1)\right]$ are axisymmetric, according to Eqs. (27b) and (27c), if the Coriolis terms on the right-hand side of Eq. (27b) are zero. At a given time, the relative importance of the Coriolis terms depends on $\Delta, \quad \alpha, \quad \bar{u}_{0}\left(=\partial \widetilde{\psi}_{0} / r \partial \alpha\right)$ and $\bar{v}_{0}\left(=-\partial \bar{\psi}_{0} / \partial r\right)$, and varies from place to place in the $(r, \alpha)$ plane. In general, the Coriolis effect is maximized at $\alpha \approx \pi / 2$ but minimized at $\alpha \approx 0$ and $\pi$ except when $\bar{v}_{0}$ is small which occurs at $t \approx 60^{\circ}$ and $90^{\circ}$. Thus the density of most of the $\widetilde{w}_{0}^{*}$ contours in Figs. 10-12 are "compressed" near $\alpha=90^{\circ}$ but "relaxed" near $\alpha=0^{\circ}$ and $180^{\circ}$.

It is also the local dependence of the Coriolis term which makes the secondary streamfunction complicated. The Coriolis terms on the right-hand side of Eq. (27a) change because the gradients of $\bar{w}_{0}$ vary from place to place, and because $\alpha$ varies. The existence of multiple cells in the normalized secondary streamfunction patterns in Figs. 10-12 are associated with the changes of the sign of the Coriolis terms in Eq. (27a). For a given case, the secondary flow is in general more complicated when $t=90^{\circ}$ since the sign of $\partial \bar{w}_{0} / \partial r$ changes more frequently at that instant. However, the complicated secondary flow at $t=90^{\circ}$ is not important to the contribution of the energy dissipations since $\left|\widetilde{\psi}_{0}\right|_{m}$ is small at that instant in comparing with $\left|\widetilde{\psi}_{0}\right|_{m}$ at other time. The secondary flow is also more complicated for larger value of $R_{\Omega}$ because of the existence of the overshoot in the radial distribution of $\widetilde{w}_{0}^{*}$ at the outer edge of the boundary layer and of the subsequent small oscillation in the essentially stagnant core. For large values of $R_{\Omega}$ (say, 1000), Fig. 12 shows that most of the deformation indeed occurs next to the pipe wall at $r=1$ for different $\Delta$ 's, which supports the asymptotic analysis for $R_{\Omega} \gg 1$ in Sec. IV.

\section{The steady stream, $\widetilde{w}_{1 s}$ and $\tilde{\psi}_{1 s}$}

The computer program for solving Eqs. (27a)-(27c) is modified here to solve Eqs. (31a)-(31c), but the resulting system of algebraic equations for Eqs. (31a)-(31c) are real. We employed the same grid systems as those in Sec. $\mathrm{V} \mathrm{C}$, and it takes $40 \mathrm{CPU}$ seconds in the CONVEX C1 computer to run a case. The computer program is checked by comparing the numerical results with the asymptotic solution for $R_{\Omega}<1$, according to Eqs. (38a)-(38d). The agreement is nice for $R_{\Omega} \leqslant 1$. For example even when $R_{\Omega}=1$ and $\Delta=1$, the maximum value of $\widetilde{w}_{1 S}$ and the minimum value of $\widetilde{\psi}_{1 S}$ together with their locations in the first quadrant are $0.01156\left(0.575,0^{\circ}\right)$ and $-0.4608 \times 10^{-4}$ $\left(0.575,45^{\circ}\right)$, according to the calculation, and $0.01203\left(0.577,0^{\circ}\right)$ and $-0.4823 \times 10^{-4}\left(0.577,45^{\circ}\right)$, according to the asymptotic solution.

Figure 13 plots the normalized axial velocity $\left(\widetilde{w}_{1 S} /\left|\widetilde{w}_{1 S}\right|_{m}\right)$ and the streamfunction $\left(\widetilde{\psi}_{1 S} /\left|\widetilde{\psi}_{1 S}\right|_{m}\right)$ patterns of the steady stream for different values of $R_{\Omega}$ and $\Delta$, where $\rfloor\left.\widetilde{w}_{1 S}\right|_{m}$ and $\left.\widetilde{\psi}_{1 S}\right|_{m}$ are the maximum values of $\left|\widetilde{w}_{1 S}\right|$ and $\left|\widehat{\psi}_{1 S}\right|$, respectively. The maximum (positive) or minimum (negative) value of $\widetilde{w}_{1 S}^{*}\left[=\widetilde{w}_{1 S} /(2 \Delta-1)\right]$ and $\widetilde{\psi}_{1 S}$ together with their locations in the first quadrant are listed next to each semicircular figure in Fig. 13. The distribution 
FIG. 13. Numerical solutions for $\tilde{w}_{1, S} /\left.\tilde{w}_{1 S}\right|_{m}$ (upper semicircle of each figure) and $\tilde{\psi}_{1 S} /\left|\tilde{\psi}_{1 s}\right|_{m}$ (lower semicircle), where $\left|\tilde{w}_{1 S}\right|_{m}$ and $\left|\tilde{\psi}_{1 S}\right|_{m}$ are the maximum values of $\left|\tilde{w}_{1 S}\right|$ and $\left|\widetilde{\psi}_{1 s}\right|$, respectively. The maximum (positive) or minimum (negative) values of $\widetilde{w}_{S S}^{*}\left[=\widetilde{w}_{1 S} /(2 \Delta-1)\right]$ and $\widetilde{\psi}_{1 S}$ together with their locations in the first quadrant are listed next to each semicircular figure. The contour values for both

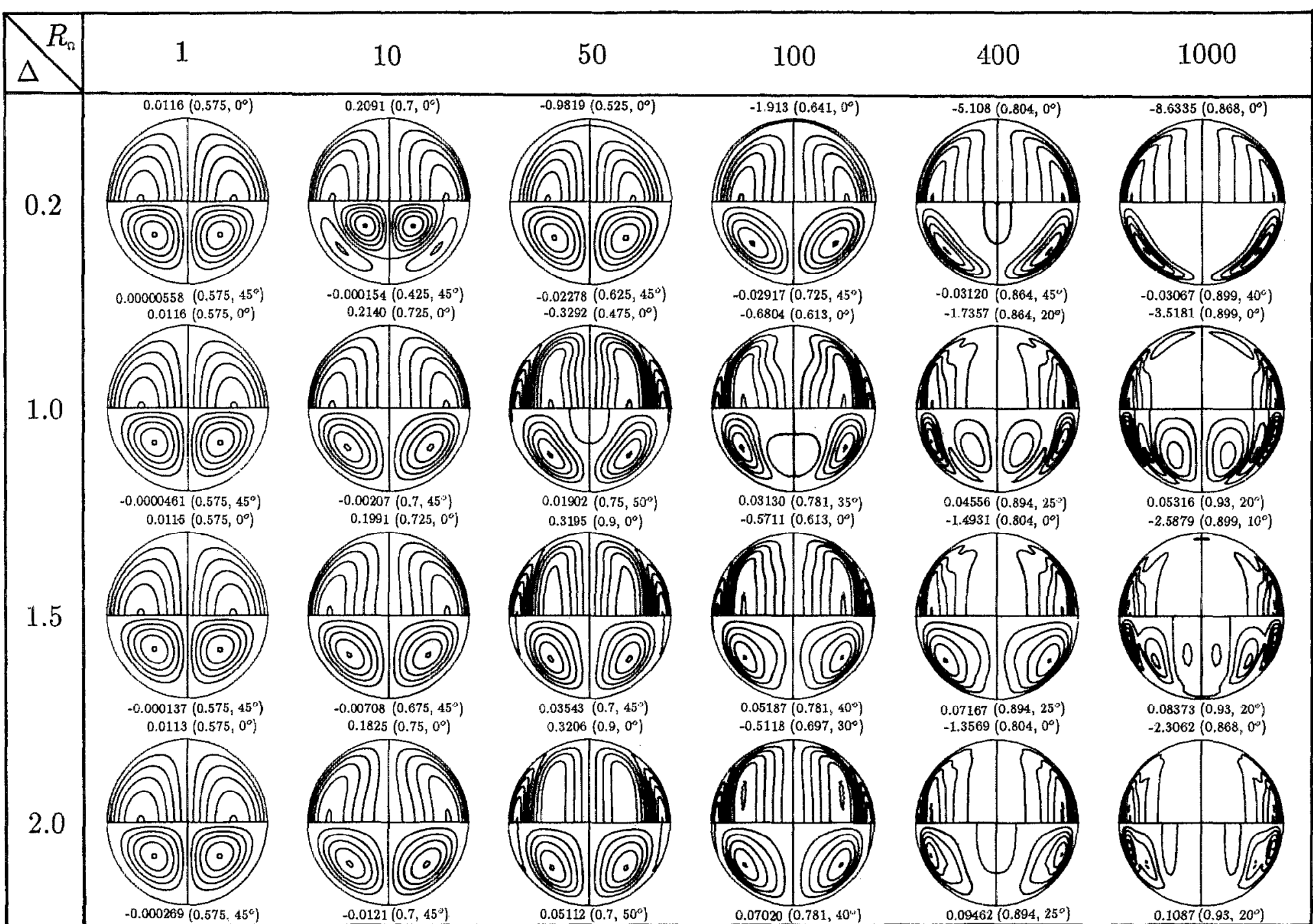

and minimum values of $\tilde{w}_{1 S}$ and $\tilde{\psi}_{1 S}$ and the result in Fig. 14 
of $\widetilde{w}_{1 S}^{*}\left[=\widetilde{w}_{1 S} /(2 \Delta-1)\right]$ along the $x$ axis $\left(x=x^{\prime} / a\right)$ are plotted in Fig. 14. Note that the scales of the vertical axes in Fig. $14\left(\widetilde{w}_{1 S}^{*}\right)$ are different for different cases. The features of the steady stream are direct consequences of the relative importance of the source terms, the viscous terms and the Coriolis terms in Eqs. (31a) and (31b) at different values of $R_{\Omega}$ and $\Delta$. The strength of the steady stream increases as both $R_{\Omega}$ (for fixed $\Delta$ ) and $\Delta$ (for fixed $R_{\Omega}$ ) increase, according to Figs. 13 and 14 . The results shown in the figures on the first column of Figs. 13 and 14 are essentially the same as the asymptotic solution for $R_{\mathbf{\Omega}} \ll 1$ according to Eqs. (38a)-(38d). However, the results change significantly as $R_{\Omega}$ increases, and such changes are quite different for different $\Delta$. The figures on each row of Fig. 14 show that the direction of the axial velocity changes as $R_{\Omega}$ increases. For a given value of $\Delta$, the direction of $\widetilde{w}_{1 S}^{*}$ at $R_{\Omega}=1$ is opposite to that at $R_{\Omega}=1000$. The direction of the secondary flow is also changed accordingly as $R_{\Omega}$ increases, which is expected since $\widetilde{w}_{1 S}$ and $\widetilde{\psi}_{1 S}$ are coupled to each other through the Coriolis terms in Eqs. (31a) and (31b). Smooth transition takes places as $R_{\Omega}$ increases, and there may exist two pairs of axial counter flows (see the case $R_{\Omega}=50, \Delta=1$ for example) and two pairs of the counter-rotating vortices for the secondary flow (see $R_{\Omega}=400, \Delta=1$ ) during the flow reversal process as $R_{\Omega}$ varies. For small $R_{\Omega}$, the steady stream (including the counter axial currents and the secondary vortices) fills the whole cross sectional area. The size of the secondary vortices reduces as $R_{\Omega}$ increases, and move toward $x= \pm 1$ where the Coriolis effect is minimized (see Fig. 13). For $\Delta>1$ and $R_{\Omega} \gg 1$, the variation of $\widetilde{w}_{1 S}$ is also confined to narrow region next to $x= \pm 1$, and $\widetilde{w}_{1 S} \approx 0$ in the core. However, although the gradient of $\widetilde{w}_{1 S}$ is steep next to the wall near $x= \pm 1$ for $R_{\Omega} \geqslant 1$ and $\Delta=0.2$, the variation of $\widetilde{w}_{1 S}$ with $x$ is approximately linear in the core (see Fig. 14).

\section{THE AXIAL OSCILLATION PROBLEM}

So far we have studied the flow driven by a threedimensional harmonic oscillation of the pipe wall [see Eq. (7e)]. Such three-dimensional oscillation problem is not easy to study experimentally. In order to understand the essential physics of the secondary flow driven by the Coriolis effect in a rotating pulsate pipe flow, it is sufficient to study the axial problem, i.e., to solve Eqs. (11a) and (11b) subjected to

$$
\psi=0, \quad \frac{\partial \psi}{\partial r}=0, \quad w=-\cos t \quad \text { at } r=1,
$$

instead of Eq. (11c). The velocity measurement for such axial oscillation problem can be studied by experiments rather easily in comparison with the three-dimensional oscillation problem. Furthermore, as we have mentioned that there are two different points between the rotating pipe flow model and the stationary pipe flow model [see the statement after Eq. (9b) in Sec. II], the study of the axial oscillation problem provides us a way to investigate the relative importance of the contributions to the energy dissipation due to these two different sources.
For the present linearized problem $(A \ll 1)$, Eqs. (20a) $-(20 \mathrm{c})$ are still valid for the axial oscillation problem except that $\widetilde{w}_{0}, \widetilde{\psi}_{0}$, and $2 \Delta-1$ are replaced by $w_{0}, \psi_{0}$, and 1 , respectively. The asymptotic results for $R_{\Omega}<1$ in Eqs. (36a), (36b), (39a), and (40), and those for $R_{\Omega}>1$ in Eqs. (44a) $-(44 c),(45 a)-(45 c)$, and (48) are valid for the axial oscillation problem if the factor $(2 \Delta-1)$ in the equations is replaced by 1 . In particular,

$$
\frac{g_{R 0}}{g_{S 0}}=1-\frac{1}{576} \Delta^{2} R_{\Omega}^{2}+O\left(R_{\Omega}^{4}\right)
$$

for $R_{\Omega}<1$, and

$$
\frac{g_{R_{\infty}}}{g_{S_{\infty}}}=G(\Delta)+O\left(R_{\Omega}^{-1 / 2}\right)
$$

for $R_{\Omega} \gg 1$, with $G(\Delta)$ shown in Fig. 6. By comparing with the three-dimensional oscillation problem, the rotating effect on the energy dissipation is moderate for the axial oscillation problem. For large $R_{\Omega}$, the rotating effect decreases the energy dissipation slightly when $\Delta<0.8$ (see Fig. 6), but increases the energy dissipation when $\Delta \geqslant 0.8$. In particular, $g_{R_{\infty}} / g_{S_{\infty}} \approx 1.34$ and 1.54 for $\Delta=1.5$ and 2 , respectively. By comparing with the differences between the axial and the three-dimensional oscillation problem, the three-dimensional oscillation of the pipe wall increases (or decreases if $\Delta<1$ ) the energy dissipation further by a factor $(2 \Delta-1)^{2}$. The numerical results in Figs. 8-14 are also the corresponding results for the axial oscillation problem provided that the factor $(2 \Delta-1)$ is replaced by unity. The asymptotic results for the steady stream, $\widetilde{w}_{1 S}$ and $\tilde{\psi}_{1 S}$, when $R_{\Omega}<1$ are obtained following similar procedures shown in Sec. IV A, they are

$$
\begin{aligned}
\psi_{1 S}= & \frac{R_{\Omega}^{5} \Delta^{2}}{35389440}\left(17 r^{2}-36 r^{4}+20 r^{6}-r^{10}\right) \sin 2 \alpha \\
& +O\left(R_{\Omega}^{7}\right) \\
w_{1 S}= & \frac{R_{\Omega}^{4} \Delta}{36864}\left(-3 r+6 r^{3}-4 r^{5}+r^{7}\right) \cos \alpha+O\left(R_{\Omega}^{6}\right), \\
u_{1 S}= & \frac{1}{r} \frac{\partial \psi_{1 S}}{\partial \alpha} \\
= & \frac{R_{\Omega}^{5} \Delta^{2}}{17694720}\left(17 r-36 r^{3}+20 r^{5}-r^{9}\right) \cos 2 \alpha \\
& +O\left(R_{\Omega}^{7}\right),
\end{aligned}
$$

and

$$
\begin{aligned}
v_{1 S}= & -\frac{\partial \psi_{1 S}}{\partial r} \\
= & -\frac{R_{\Omega}^{5} \Delta^{2}}{17694720}\left(17 r-72 r^{3}+60 r^{5}-5 r^{9}\right) \sin 2 \alpha \\
& +O\left(R_{\Omega}^{7}\right) .
\end{aligned}
$$

The axial oscillation problem can be transformed into a rotating straight pipe flow driven by an imposed pulsat- 


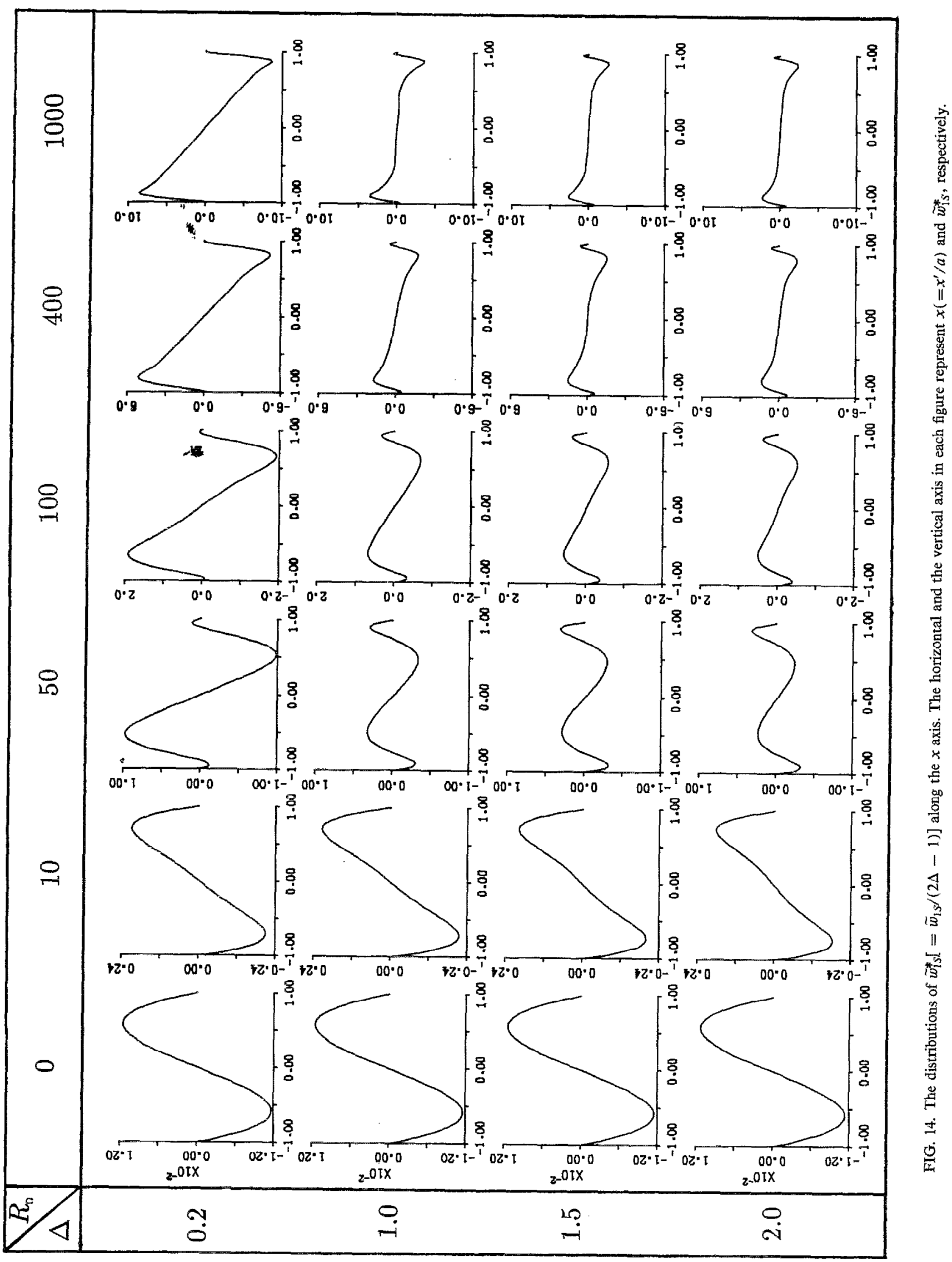


ing axial pressure gradient with the pipe wall held fixed via an unsteady Galilean transformation (see Panton, ${ }^{14}$ Chap. 10). Such resulting pulsating flow is similar to the pulsating flow in a stationary curved pipe studied by Lyne ${ }^{15}$ and Zalosh and Nelson ${ }^{16}$ that (1) both problems possess secondary flows in the plane perpendicular to the imposed axial pressure gradicnt; (2) stcady streams can be gencrated via the nonlinear convection effect in both problems, and (3) both problems are governed by threedimensionless parameters: $R_{\Omega}, \Delta$, and $A$ in the present rotating straight pipe flow, and $\bar{\alpha}, \delta$, and $\bar{A}$ for the stationary curved pipe flow according to Zalosh and Nelson. Here $\bar{\alpha}=a(\Omega / v)^{1 / 2}, \delta=a / R$, and $\bar{A}=(a / R)(K a / \Omega v)^{2}$, where $R$ is the radius of curvature of the curved pipe, and $K$ and $\Omega$ are the amplitude and frequency, respectively, of the pulsating axial pressure gradient. Zalosh and Nelson studied the problem when $\delta<1$ and $\bar{A}<1$ with arbitrary $\bar{\alpha}$. Lyne studied the problem when $\delta<1$ and $\bar{\alpha}>1$ with either $\bar{A} \ll 1$ or $\bar{A} \gg 1$. The parameters $R_{\Omega}, \Delta$, and $A$ in the rotating pipe flow play the role of $\bar{\alpha}^{2}, \delta$, and $(\bar{A} / \delta)^{1 / 2} / \bar{\alpha}^{2}$ in the curved pipe flow. Here we study the rotating straight pipe flow for arbitrary values of $R_{\Omega}(=0.1-1000)$ and $\Delta(=0-2)$ but small values of $A$. The result for $\Delta=0.2$ in Figs. 10-14 can be compared qualitatively with the result in Zalosh and Nelson and the result for $\bar{A}<1$ in Lyne. An interesting feature of the pulsating curved pipe flow is that the secondary flow in the core directs towards the center of curvature, which is constrast to the steady unidirectional flow in curved pipes. The result for $\Delta=0.2$ at $t=0^{\circ}$ in Fig. 11 also indicates similar behavior that the secondary flow in the core directs towards the opposite direction to that in unidirectional rotating pipe flow (see Lei and $\mathrm{Hsu}^{12}$ ). However, there exist fundamental differences between the rotating straight pipe flow and the stationary curved pipe flow that: (1) $\delta$ is always less than unity but $\Delta$ may be greater than unit. In practical applications, $\delta=O\left(10^{-1}\right)$ but $\Delta=O(1)$. As the relative magnitude of the secondary flow to the primary flow is of order $\delta$ for the stationary curve pipe flows and of order $\Delta$ for the rotating straight pipe flow, the secondary flow could be of the same order as the primary flow for the rotating straight pipe flow. (2) The primary flow and the secondary flow are coupled together through the convective and Coriolis effects for the rotating straight pipe flow, but only through the convective effect for the stationary curved pipe flow for $\delta<O\left(10^{-1}\right)$. Thus the details of the flow patterns vary significantly between the present rotating straight pipe flow and that in stationary curved pipe flow in Zalosh and Nelson.

\section{CONCLUSION}

The incompressible laminar pulsatile flow in a rotating circular straight pipe is governed by three dimensionless parameters: the wobble Reynolds number $R_{\Omega}$, the frequency ratio $\Delta$, and the amplitude parameter $A$. Here the rotation axis is perpendicular to the pipe axis, and at a distance from the pipe axis which is much greater than the radius of the pipe. This rotating pulsate pipe flow is driven by a three-dimensional harmonic oscillation of the pipe wall, and is a model proposed in this paper for studying the spin synchronous mode fluid motion inside a nutation fluid damper on board of a spinning satellite. The present paper studies the detailed quasisteady behavior of the flow field for small values of $A$, with particular emphasis on the energy dissipation due to the relative fluid motion. Asympototic analyses have been carried out for $R_{\Omega} \leqslant 1$ and $R_{\Omega}>1$. It is found that the rotation has a significantly effect on the energy dissipation. Let $g$ be a normalized energy dissipation per cycle of the pipe wall oscillation. As $\Delta$ increases from $0, g$ decreases monotonically from $g_{S}$ ( $g \equiv g_{S}$ when $\Delta=0$ ), equal to 0 when $\Delta=0.5$, and then increases as $\Delta$ increases. For $\Delta>1, g / g_{S}$ increases roughly with $(2 \Delta-1)^{2}$. In particular, $g / g_{S} \approx 5.36$ for $\Delta=1.5$ and $g / g_{S} \approx 13.84$ for $\Delta=2$, in comparison with $g / g_{S}=1$ for all $\Delta$, according to the previous stationary pipe flow model in literature. Previous expcrimental results for studying the damping of the nutation angle of a gyroscopic system with a nutation fluid damper showed that $g / g_{S} \approx 4.35$ and 5.43 for $\Delta=1.5$ and 2 , respectively. Thus the present rotating pipe flow model provides a better prediction for estimating the energy dissipation rate in comparison with the previous stationary pipe flow model. Numerical solution has also been carried out to check the asymptotic analysis, which bridges the above two asymptotic regimes when $R_{\Omega}$ is of intermediate value. Detailed flow structures have been investigated for $R_{\Omega}=0.1-1000$ and $\Delta=0-2$ for small $A$. It is also found that a steady stream can be induced by the nonlinear convective effect for finite values of $A$. The flow pattern of such steady stream consists of pairs of counter axial flows and counter rotating vorticies, and varies significantly for different values of $R_{\Omega}$ and $\Delta$.

The results for $g$ discussed above are based on $A=0$. Although Eq. (34b) shows that the effect of $A$ on $g$ is small when $A$ is small, it is not clear how the effect of $A$ on $g$ when $A=O(1)$. The flow when $A=O(1)$ can be studied numerically by solving Eqs. (15a)-(15c). Such numerical study has been carried out and will be reported in a succeeding paper. It is found that for given values of $R_{\Omega}$ and $\Delta, g$ increases as $A$ increases, reaches a maximum value when $A \approx 1$, and then drops substantially as $A$ increases further.

There are two mechanisms which produce additional energy dissipation for the present rotating pipe flow model in comparison with the previous stationary pipe flow model: (1) the secondary flow induced by the Coriolis effect, and (2) the three-dimensional pipe wall oscillation. By studying the problem driven only by an axial harmonic oscillation of the pipe wall, it is found that although the first mechanism increases the energy dissipation substantially for $\Delta=1.5$ and 2 , the second effect is even more important, which increases the energy dissipation by a factor $(2 \Delta-1)^{2}$ for $\Delta>1$.

\footnotetext{
${ }^{1}$ G. F. Carrier and J. W. Miles, "On the annular damper for a freely precessing gyroscopic," J. Appl. Mech. 27, 237 (1960).

${ }^{2}$ W. F. Cartwright, E. C. Massingill, and R. D. Trueblood, "Circular constraint nutation damper analysis," Report No. TM 62-205, GM DRL Dept., General Motor Corp., Detroit, Michigan, 1961.

${ }^{3}$ K. T. Alfriend, "Partially filled viscous ring nutation damper," J. Spacecr. Rockets 11, 456 (1974).
} 
${ }^{4}$ W. F. Cartwright, E. C. Massingill, and R. D. Trueblood, "Circular constraint nutation damper," AIAA J. 1, 1375 (1963).

${ }^{5}$ P. G. Bhuta and L. R. Koval, "A viscous ring damper for a freely precessing satellite," Int. J. Mech. Sci. 8, 383 (1966).

${ }^{6} \mathrm{~J}$. W. Miles, "On the annular damper for a freely precessing gyroscope-II," J. Appl. Mech. 30, 189 (1963).

7J. A. Hraster, Memorandum to H. C. Hoffman, November 29, 1972, NASA/Goddard Space Flight Center, Greenbelt, MD.

${ }^{8} \mathrm{~K}$. T. Alfriend, "Analysis of a partially filled viscous ring damper-final report," Report No. NGR-33-010-169, October 1973, Cornell University, Ithaca, NY.

${ }^{9}$ E. B. V. Dussan, "On the spreading of liquids on solid surfaces: static and dynamic contact lines," Annu. Rev. Fluid Mech. 11, 371 (1979).
${ }^{10}$ S. A. Berger, L. Talbot, and L. S. Yao, "Flow in curved pipes," Annu. Rev. Fluid Mech. 15, 461 (1983).

${ }^{11} \mathrm{H}$. Ito and $\mathrm{K}$. Nanbu, "Flow in rotating straight pipes of circular crosssection," J. Basic Eng. 93, 383 (1971).

${ }^{12}$ U. Lei and C. H. Hsu, "Flow through rotating straight pipes," Phys. Fluids A 2, 63 (1990)

${ }^{13} \mathrm{C}$. F. Ho, "Pulsatile flow in a rotating straight pipe," Master thesis, National Taiwan University, 1988.

${ }^{14}$ R. L. Panton, Incompressible Flow (Wiley, New York, 1984).

${ }^{15}$ W. H. Lyne, "Unsteady viscous flow in a curved pipe," J. Fluid Mech. 45, 13 (1970).

${ }^{16}$ R. G. Zalosh and W. G. Nelson, "Pulsating flow in curved tube," J. Fluid Mech. 59, 693 (1973). 
Copyright $\odot 2003$ EBSCO Publishing 